\title{
Frequency Domain Analysis and Equalization for Molecular Communication
}

\author{
Yu Huang, Fei Ji, Member, IEEE, Zhuangkun Wei, Miaowen Wen, Senior Member, IEEE, \\ Xuan Chen, Yuankun Tang, and Weisi Guo, Senior Member, IEEE
}

\begin{abstract}
Molecular Communication (MC) is a promising micro-scale technology that enables wireless connectivity in electromagnetically challenged conditions. The signal processing approaches in MC are different from conventional wireless communications as molecular signals suffer from severe inter-symbol interference (ISI) and signal-dependent counting noise due to the stochastic diffusion process of the information molecules. One of the main challenges in MC is the high computational complexity of the existing time-domain ISI mitigation schemes that display a third-order polynomial or even exponential growth with the ISI length, which is further exasperated under the high symbol rate case. For the first time, we develop a frequencydomain equalization (FDE) with lower complexity, capable of achieving independence from the ISI effects. This innovation is grounded in our characterization of the channel frequency response of diffusion signals, facilitating the design of receiver sampling strategies. However, the perfect counting noise power is unavailable in the optimal minimum mean square error (MMSE) equalizer. We address this issue by exploiting the statistical information of the transmit signal and decision feedback for noise power estimation, designing novel MMSE equalizers with low complexity. The FDE for MC is successfully developed with its immunity to ISI effects, and its signal processing cost has only a logarithmic growth with symbol length in each block.
\end{abstract}

Index Terms-molecular communication, frequency response, sampling, frequency domain equalization, signal-dependent noise.

\section{INTRODUCTION}

A Wide range of organisms use the molecules as the information carrier for message transmission, and this mechanism is known as molecular communication (MC) from a communication perspective [1]. MC is recognized as not only an alternative for conventional communication techniques, but

The work of Y. Huang, F. Ji, X. Chen, Y. Tang, and M. Wen was supported in part by the Key Program of Marine Economy Development (Six Marine Industries) Special Foundation of Department of Natural Resources of Guangdong Province (GDNRC [2020]009), in part by the National Natural Science Foundation of China under Grant 61871190, in part by the Natural Science Foundation of Guangdong Province under Grant 2018B030306005, and in part by the Fundamental Research Funds for Central Universities under grant 2019SJ02. The work of Y. Huang is funded by China Scholarship Council No. 201906150032. (Corresponding author: Miaowen Wen.)

Y. Huang is with the School of Electronics and Information Engineering, South China University of Technology, Guangzhou 510641, China, and also with the School of Aerospace, Transport and Manufacturing, Cranfield University, Bedford MK43 0AL, U.K. (e-mail: ee06yuhuang@mail.scut.edu.cn).

F. Ji, M. Wen, X. Chen, and Y. Tang are with the School of Electronics and Information Engineering, South China University of Technology, Guangzhou 510641, China (e-mail: eefeiji@scut.edu.cn; eemwwen@scut.edu.cn; eechenxuan@mail.scut.edu.cn; eeyktang@mail.scut.edu.cn).

Z. Wei is with the School of Engineering, University of Warwick, Coventry CV4 7AL, U.K. (e-mail: zhuangkun.wei@warwick.ac.uk).

W. Guo is with the School of Aerospace, Transport, and Manufacturing, Cranfield University, Milton Keynes MK43 OAL, U.K., and also with the Alan Turing Institute, London NW1 2DB, U.K. (e-mail: weisi.guo@cranfield.ac.uk). also a promising candidate for future applications, such as nanonetwork and bio-molecular sensing [2]. MC has been comprehensively reviewed in [3], and most of its research fall within the time domain.

The information is generally modulated via molecular concentration and type, yielding for example, concentration shift keying (CSK) and molecular shift keying (MoSK) [4], [5]. Note that the on-off keying (OOK) is an energy-efficient modulation scheme, regarded as a special case of CSK, where both the release and silence states are used to convey information [6]. As for the variants of MoSK, the isomer based ratio shift keying exploits the ratio between different types of molecules as the information source [7]; the generalized MoSK achieves a higher information rate by employing the combination of different types in each symbol duration, avoiding the single-type transmission constraint in MoSK [8]. Moreover, the molecular type permutation shift keying uses the full permutations of different types of molecules to transfer information [9]. In addition to the conventional singleinput single-output systems, the multiple-input multiple-output (MIMO) techniques can be used in MC [10]-[12], where the diversity and multiplexing modes achieve better error performance and higher transmission rates, respectively. To overcome the inter-link interference (ILI) brought by the spatial multiplexing mode, one of the index modulation family members, i.e., spatial modulation, has been proposed for ILI mitigation [13], [14]. Moreover, the state-of-the-art modulation techniques for MC have been thoroughly reviewed in [15]. After modulation at the transmitter, the released molecules undergo the stochastic diffusion process in the channel, which promises the energy-efficient feature of the MC system; however, molecules' random movement makes their arrivals at the receiver unpredictable. Hence, the previously transmitted symbols may overlap with the current symbol, bringing the intersymbol interference (ISI) that severely degrades the receiver's detection performance [16]. Against this background, the ISI mitigation is essentially one of the most challenging issues in the MC system, and both transmitter and receiver strive to achieve this goal.

Though CSK is credited for its simple realization, it suffers from severe ISI effect due to the accumulation of information molecules of the same type. On the other hand, although MoSK has the robustness to the ISI, it imposes high complexity on the transceiver design as more than one types of chemical reservoirs and sensors are of necessity. In addition to the modulation schemes, the receiver side also plays an essential role in dealing with interference. Despite the primitive symbol detector with a fixed threshold, many detection schemes consider the ISI effect. The sequence detection based on the 
optimal maximum a posteriori (MAP) and maximum likelihood (ML) criteria exhibit the optimal performance, while its high computational complexity is prohibitively expensive to implement in nano-scale transceivers. Concerning the tradeoff between complexity and detection performance in $\mathrm{MC}$, a low-complexity near ML sequence detector was proposed in [17], and the time-domain equalization (TDE) was used to combat ISI as it requires much lower computational complexity, while sub-optimal performance is obtained [18]. However, the computational complexity of TDE is proportional to the number of channel taps. Consequently, the implementation of TDE is computationally cumbersome when the transmission rate is high. In this case, such equalizers become ineffective due to the complexity constraint.

This paper proposes frequency-domain equalization (FDE) instead of TDE for MC to solve the aforementioned problems. Nevertheless, to the best of the authors knowledge, there is no study manifesting the explicit form of the frequencydomain characteristics for MC. To this end, first, we perform the frequency-domain analysis for MC channels to lay the foundation for the FDE. Then, we derive frequency response, signal energy, bandwidth, and sampling frequency from the frequency-domain analysis, based on which a low-complexity FDE structure is proposed for MC with high symbol rate transmission. In summary, the main contributions of this paper are as follows.

- The frequency response of CIR is derived for the diffusion-based MC channel, manifesting its low-pass nature. As a result, the CIR is proved to have finite energy in the frequency or time domain.

- The definitions of bandwidth and sampling strategies for the one-shot CIR are proposed.

- The proposed FDE system in MC with the signaldependent counting noise is proved to be the singlecarrier FDE in nature. Despite having lower complexity than its TDE counterparts, it only requires a single type of information molecules and a single sample for every symbol duration.

- In addition to the linear zero-forcing $(\mathrm{ZF})$ equalizer, the optimal minimum mean square error (MMSE) equalizer in the presence of counting noise cannot be deployed as its perfect noise power in each block is unattainable. Against this background, the sub-optimal MMSE equalizers are proposed, relying on the noise power estimation from the statistical information of the transmit signal and the decision feedback (DF) of the detected symbols.

The remainder of this paper is organized as follows. Section II retrospects the related works of frequency-domain analysis and equalization in the MC system, and Section III reviews the MC signal characteristics in the time domain. In Section IV, we present frequency-domain analysis for MC signals, where the frequency response, energy in the frequency domain, concepts of bandwidth, and sampling frequency in MC are derived. Section V proposes the FDE with ZF and MMSE criteria for the MC system. After the analytical studies, Section VI demonstrates the numerical results. Finally, the conclusion of this work is drawn, and the future research direction is foreseen in Section VII.

\section{RELATED WORKS}

Most equalizers in the literature are studied from the timedomain perspective. The linear TDE based on the MMSE criterion was proposed for MC with OOK [18], where only one received sample in each symbol duration is considered. The TDE becomes nonlinear with the previously detected bits being the input, known as the decision-feedback equalizer (DFE), which performs better than its linear counterpart at the cost of computational complexity. The work of [12] extended the time-domain DFE with $\mathrm{ZF}$ and MMSE criteria to the MIMO MC system with OOK. The combination of the timedomain DFE and Volterra series formed a Volterra-DFE [19], which performs better than the conventional DFE. However, the computational complexity of the Volterra-DFE grows exponentially with the Volterra series order, which is prohibitively large for the nano-scale MC. The fractionally-spaced TDE has a similar problem as multiple samples are used within each symbol duration for better detection performance [20], which in turn requires higher computational complexity at the receiver side. A linear time and space-time equalizers were proposed for spatial modulation in MC [21]. For MoSK, TDEs with matched filter, $\mathrm{ZF}$, and MMSE criteria were proposed to eliminate the ISI at the receiver side [22]. Despite the prevalence of TDE, the concept of FDE emerges in $\mathrm{MC}$ recently [23], [24]. The FDE based on the MMSE criterion was studied in [23], while its ZF counterpart was analyzed in [24]. However, these FDE-related works assumed low-pass characteristics of the MC channel without providing rigorous proof, and the noise model considered the additive white Gaussian noise (AWGN) like those in conventional wireless communications. To the best of our knowledge, there are neither explicit results nor rigorous frequency-domain analysis for MC. Furthermore, no FDE design has been studied for the diffusion-based $\mathrm{MC}$ with the non-stationary counting noise that depends on the current signal strength.

The first MC work that concerns the frequency domain was the end-to-end system research in [25]. The Fourier transform of CIR provided an intuitive explanation of the system transfer function as multiplication operation rather than convolution operation is used, and the bandwidth was quantified via numerical results in [25]. Following the time-frequency analysis of the end-to-end model in [25], the noise characteristics were studied in [26]. Moreover, the pulses with different frequencydomain characteristics were compared in [27], and spikelike pulse with short interval was regarded as a satisfactory shaping strategy through the numerical simulation. In [28], the transfer function of the MC channel with only diffusion effect was derived, based on which the pulse shaping strategy was designed via the inverse Laplace transform.

The frequency-domain analysis can give new insights into the impact of the frequency components. In addition to the amplitude and type, MC also adopted the frequency shift keying (FSK) to transmit information [4], as such mechanism naturally exists in the natural calcium signaling [29]. As the enzymatic circuit displays the frequency selective behavior, 
it was envisioned as the FSK decoder for MC [30]. An oscillating transmitter generated different longitudinal waves, realizing the possibility of bandpass modulation in MC [31], where the frequency demultiplexing was successfully achieved via recovering the modulated signal from the multiplexed signal. Furthermore, inspired by the optical communication technique, the orthogonal frequency division multiplexing (OFDM) concept was presented for MC in [32]. The inverse fast Fourier transform modulates the signal in OFDM based $\mathrm{MC}$, and the frequency selective channel can be transformed to the parallel channel, such that the ISI can be completely removed with a proper length of the cyclic prefix (CP). The simulated frequency response of a diffusion-based MC signal showed that the low-frequency components contain most of the signal energy [33], where the rectangular wave was used as the input signal. In light of the coronavirus disease (COVID19) pandemic, viral aerosol transmission and detection models were used to study the behavior of virus spread in the context of communication [34]. Unlike conventional MC, specific initial and boundary conditions were assumed in [34] to derive the frequency response, which was recognized to facilitate the synchronization and the choice of sampling frequency. The robustness of the frequency modulation in a calcium signaling system against noises was studied in [35], where the fast Fourier transform converts the time-domain received signal to its frequency-domain counterpart, and the spectral analysis offers an intuitive explanation on the noise impact.

Overall, frequency-domain analysis plays a significant role in $\mathrm{MC}$ research, while there is no available literature related to its rigorous derivation, and the implementation of FDE in $\mathrm{MC}$ is based on the frequency analysis.

\section{TIME DOMAIN System ModeL}

The MC system consists of three fundamental parts, i.e., transmitter, channel, and receiver. The transmitter first sends the information by releasing molecules into the channel, governed by the diffusion mechanism. Finally, the receiver samples the molecular signal and then decodes it via detection methods. For intuitive explanation, the block diagram of an end-to-end MC system is depicted in Fig. 1.

In this paper, the molecular signal is represented by the number/concentration of information molecules. The transmitter is regarded as a point source located in the origin of the coordinate, a three-dimensional (3D) communication channel with infinite space is considered, and the receiver has a spherical shape with radius $r$.

\section{A. Free Diffusion Channel}

The collision of molecules causes their random movement, known as the Brownian motion or diffusion, and such diffusion dominates in the micro-scale environment. The diffusion effect is assumed to be inevitable in this paper. The diffusion coefficient determines the dispersion scale of diffusion and has the following form as

$$
D=\frac{k_{B} T}{6 \pi \eta R}
$$

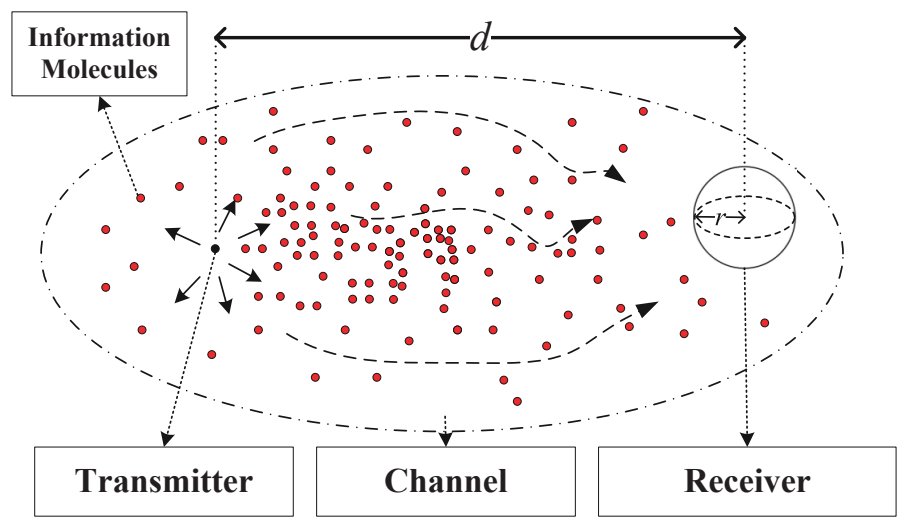

Fig. 1. Diagram of an end-to-end MC system.

where $k_{B}=1.38 \times 10^{-23} \mathrm{~J} / \mathrm{K}$ is the Boltzmann's constant, $T$ refers to the temperature, $\eta$ is the dynamic viscosity of the fluid, and $R$ represents the radius of the information molecule [36]. When only the diffusion mechanism is considered, such a channel is referred to as the free diffusion channel, where the spatio-temporal distribution of the average molecular concentration obeys Fick's second law as

$$
\frac{\partial c(\mathbf{d}, t)}{\partial t}=D \nabla^{2} c(\mathbf{d}, t)
$$

where $c(\mathbf{d}, t)$ is the mean molecular concentration at coordinate $\mathbf{d}$ and time $t$, and $\nabla^{2}$ is the Laplace operator, given by $\nabla^{2}=\frac{\partial^{2}}{\partial x^{2}}+\frac{\partial^{2}}{\partial y^{2}}+\frac{\partial^{2}}{\partial z^{2}}$ in the three dimensional Cartesian coordinates. When an impulsive transmission is released by transmitter at time $t=0$, the initial condition is given by

$$
c(\mathbf{0}, t \rightarrow 0)=Q \delta(\mathbf{d}),
$$

where $Q$ is the number of the information molecules per impulse, and $\delta(\cdot)$ is the Dirac function. Besides, the concentration vanishes at the infinitely far distance from the transmitter, which corresponds to the boundary condition as

$$
c(\|\mathbf{d}\| \rightarrow \infty, t)=0 .
$$

When the receiver is situated at $\mathbf{d}$, and $\|\mathbf{d}\| \gg r$, it has uniform concentration inside [37]. Based on the preceding initial and boundary conditions in (3) and (4), the molecular concentration in (2) yields

$$
c(\mathbf{d}, t)=\frac{Q}{(4 \pi D t)^{\frac{3}{2}}} \exp \left(-\frac{\|\mathbf{d}\|^{2}}{4 D t}\right) .
$$

By solving the partial derivative of (5) with respect to time to zero, i.e.,

$$
\frac{\partial c(\mathbf{d}, t)}{\partial t}=0
$$

the peak concentration arrival time in the free diffusion channel is derived as

$$
t_{p}=\frac{\|\mathbf{d}\|^{2}}{6 D} .
$$




\section{B. Received Signal}

In this paper, the CIR is defined as the impact of a single information molecule on the receiver, which can be deemed as a scaled version of the concentration signal with bit-1 transmission. When both transmitter and receiver are immobile and the channel environment is steady, we have fixed $\mathbf{d}$ and constant $D$. Then, under the assumption that $\|\mathbf{d}\| \gg r$, the CIR with respect to time is defined as

$$
h(t)=\frac{c(\mathbf{d}, t)}{Q}=\gamma \frac{\exp \left(-\frac{\alpha}{t}\right)}{t^{\frac{3}{2}}},
$$

where we have

$$
\alpha=\frac{\|\mathbf{d}\|^{2}}{4 D}, \quad \gamma=\frac{1}{(4 \pi D)^{\frac{3}{2}}},
$$

for ease of exposition in the following derivation.

When OOK is employed, let $\mathbf{b}$ denote the transmit binary data block with length $M$ as follows,

$$
\mathbf{b}=\left[b_{0}, \ldots, b_{m}, \ldots, b_{M-1}\right]^{\mathrm{T}},
$$

where the value of $b_{m}$ is either 0 or 1 , and $[\cdot]^{\mathrm{T}}$ denotes the transpose operation. In OOK, the transmitter emits $Q$ information molecules in an impulsive manner for bit-1, while it stays silent for bit- 0 . Thus, the transmitted signal has the form of

$$
x(t)=\sum_{m=0}^{\min \left\{\left\lfloor\frac{t}{T_{b}}\right\rfloor, M-1\right\}} b_{m} Q \delta\left(t-m T_{b}\right),
$$

where $\lfloor\cdot\rfloor$ is the floor function, and $T_{b}$ refers to the binary symbol duration. Given the CIR $h(t)$, the corresponding noiseless received signal at time $t$ has the form of

$$
y(t)=h(t) \otimes x(t),
$$

where $\otimes$ denotes the convolution operation. With the introduction of noise, the noisy signal at time $t$ can be expressed as

$$
z(t)=y(t)+n(t)
$$

where $n(t)$ is the noise at time $t$ that is typically known as the counting noise [26], following the normal distribution with zero mean and signal-dependent variance as

$$
n(t) \sim \mathcal{N}\left(0, \frac{y(t)}{V_{\mathrm{R}}}\right),
$$

where $V_{\mathrm{R}}=\frac{4}{3} \pi r^{3}$ is the volume of the spherical receiver.

Figure 2 shows the trend of noiseless molecular concentration signal with respect to time. The concentration signal rapidly increases before reaching its peak value in the initial stage, and it then slowly descends, vanishing after a long duration. For comparison, in addition to the noiseless version, the noisy signal with counting noise in (14) is also shown in Fig. 2.

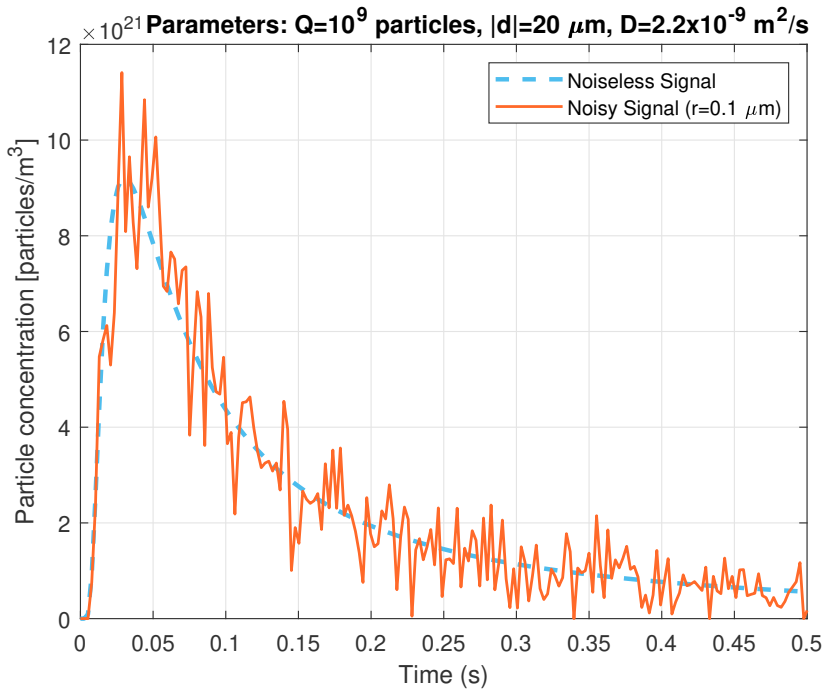

Fig. 2. Noiseless and noisy concentration signals in the free diffusion channel.

\section{Frequency AnAlysis}

The previous section reviews the molecular concentration signal via diffusion from the time domain, while this section concerns its frequency-domain analysis. Channel frequency response is the Fourier transform of the time-domain CIR, which plays an important role in communication systems. The channel energy is obtained over the whole frequency range, and the definition of bandwidth as well as sampling frequency is proposed.

\section{A. Frequency Response}

Proposition 1: $h(t)$ is an absolutely integrable function when both transmission distance $\|\mathbf{d}\|$ and diffusion coefficient $D$ are finite, and the absolute integration of CIR is given by

$$
\int_{-\infty}^{\infty}|h(t)| d t=\frac{1}{4 \pi\|\mathbf{d}\| D} .
$$

Proof: See Appendix A.

Thus, $h(t)$ has its Fourier transform, which is typically referred to as the frequency response for free diffusion channels, given by

$$
\begin{aligned}
H(\omega) & =\int_{-\infty}^{\infty} h(t) \exp (-j \omega t) d t \\
& =\frac{1}{4 \pi\|\mathbf{d}\| D} \exp \left(-\|\mathbf{d}\| \sqrt{\frac{j \omega}{D}}\right) .
\end{aligned}
$$

Proof: See Appendix B.

Given (16), the amplitude of the frequency response is

$$
|H(\omega)|=\frac{1}{4 \pi\|\mathbf{d}\| D} \exp \left(-\|\mathbf{d}\| \sqrt{\frac{\omega}{2 D}}\right),
$$

and the phase component is

$$
\angle H(\omega)=-\|\mathbf{d}\| \sqrt{\frac{\omega}{2 D}} .
$$


Remark 1: The results in (17) and (18) show that both the amplitude and phase depend on the channel parameters $\|\mathbf{d}\|$ and $D$.

Remark 2: For $\omega \geq 0$, the amplitude and phase of the frequency response monotonically decrease with the frequency component $\omega$. The diffusion-based MC system is, thus, a lowpass channel in the frequency domain.

\section{B. Energy of CIR}

The energy spectrum density of the desired signal is defined as

$$
S_{c}(\omega)=|H(\omega)|^{2},
$$

whose integral over the frequency is the signal energy in the form

$$
\frac{1}{2 \pi} \int_{-\infty}^{\infty} S_{c}(\omega) d \omega=\frac{1}{16 \pi^{3}\|\mathbf{d}\|^{4} D}
$$

Proof: See Appendix C.

The Parseval's theorem states that the frequency-domain energy equals the time-domain energy. Against this background, an alternative way is to obtain the energy in the time domain as below

$$
\frac{1}{2 \pi} \int_{-\infty}^{\infty}|H(\omega)|^{2} d \omega=\int_{-\infty}^{\infty}|h(t)|^{2} d t .
$$

Note that the right-hand side of (21) can be derived via similar methods in Appendix A, and an equivalent result as the righthand side of (20) can be obtained.

Remark 3: The CIR of the MC has finite energy according to (20), and it is inversely proportional to the diffusion coefficient, and the fourth power of transmission distance.

\section{Bandwidth and Sampling Frequency}

The concentration signal is originally continuous in time, while the digital communication system typically deals with the discrete signal. A discrete sample sequence with a proper sampling frequency is necessary to recover an analog signal with a finite bandwidth.

Here, the sampling frequency for one-shot CIR is studied from a frequency-domain perspective. Since the frequency response of CIR in MC channel shows an exponentially decay over the whole frequency range, the first step is to quantify the cutoff frequency that limits the frequency range, and the signal energy of the truncation over the frequency response from the direct current component $(0 \mathrm{~Hz})$ to the cutoff frequency, i.e., bandwidth, should contain most of the energy over the whole frequency range.

We consider the one sided energy spectrum as follows

$$
\int_{0}^{\omega_{c}}|H(\omega)|^{2} d \omega=\eta \int_{0}^{\infty}|H(\omega)|^{2} d \omega,
$$

where $\omega_{c}$ is called $\eta$ fractional power containment bandwidth [38], and $\eta$ represents the portion of the total signal energy, whose value is in the interval of $(0,1)$. Typically, $\eta=0.99$, meaning that $99 \%$ of the signal power is inside the occupied band.
For the free diffusion channel, we have

$$
\omega_{c}=\frac{D\left(W_{-1}\left(\frac{\eta-1}{\exp (1)}\right)+1\right)^{2}}{2\|\mathbf{d}\|^{2}},
$$

where $W_{k}(\cdot)$ is the $k$-th branch of the Lambert $\mathrm{W}$ function [39].

Proof: See Appendix D.

According to the Nyquist theorem, the sampling frequency should satisfy

$$
\frac{\omega_{c}}{\pi} \leq F_{s}<\infty .
$$

Note that the result given in (24) is for the information recovery of a one-shot concentration signal, which can be used for channel and distance estimation applications [40].

\section{Frequency Domain Equalization}

Equalization mitigates the ISI from the previously transmitted symbols, and the TDE has been intensively studied in MC. However, the computational complexity of the TDE is proportional to the number of channel taps, i.e., the length of ISI, or equivalently, the symbol rates. Thus, severe ISI impact is imposed on the MC system with high transmission rate, while the high computational complexity hinders the implementation of TDE due to the device constraint in the nano-scale receiver. An alternative approach to overcome this issue is the FDE via block-wise transmission, which possesses lower computational complexity than TDE. The spectrum in the conventional electromagnetic wave based communication systems is typically regulated by the authorities to avoid interference, while there is no such concern for the MC system. Against this background, MC can fully occupy a proper bandwidth without any division.

As a consequence, the FDE in $\mathrm{MC}$ is inherently a singlecarrier FDE (SC-FDE) [41], which has been thoroughly studied in the context of wireless communication. For this reason, this section proposes the SC-FDE structure, and its realization process in MC. Given the continuous-time signal in (13), its frequency-domain representation is

$$
Z(\omega)=H(\omega) X(\omega)+N(\omega),
$$

where $Z(\omega), X(\omega)$, and $N(\omega)$ are the frequency responses of the received signal, the transmit signal, and noise, respectively.

\section{A. Structure of SC-FDE}

Yet, the realistic MC system processes the discrete-time signal, and so does the SC-FDE in MC. The synchronization remains a specific research issue in MC systems which has been studied thoroughly in the works such as [42]-[46]. Note that this paper assumes perfect synchronization. Besides, the perfect channel state information (CSI) is typically assumed to be known at the receiver for performance evaluation, while it has to be estimated and tracked in practice. The channel estimation is also a vital research topic in MC. So far, there has been some works concerning this issue [12], [47]-[49]. The system structure of SC-FDE in MC is illustrated in Fig. 3, which is further elaborated as follows. 
First, the transmitted data is mapped according to the modulation scheme (OOK), and the original data block has a length of $M$. The guard interval (GI) with length $G$ is inserted to mitigate the inter-block interference (IBI), which is designed according to the number of channel taps $L$. This indicates that the previously transmitted $I=L-1$ symbols can affect the detection of the current symbol, known as the ISI effect. When the length of GI is longer than the length of ISI $I$, IBI can be completely canceled. Consequently, the new data block contains both the transmit symbol and redundant GI, which can be delivered via the molecular representation, known as the digital to molecular (D/M) conversion for emission. The molecules then propagate through the channel towards the receiver, which samples the signal in each symbol duration. The continuous-time molecular signal is transformed into the discrete-time digital form via the sampling process, i.e., molecular to digital (M/D) conversion. After the removal of GI, the discrete Fourier transform (DFT) converts the timedomain signal to the frequency domain, and the FDE is implemented based on certain criteria. In response to the FDE output, the inverse DFT (IDFT) transforms it back to the time domain, and finally, the detection technique can be used to recover the data stream.

\section{B. Discrete-time Signal}

For practical considerations, the signal detection process at the receiver does not follow the sampling frequency derived in Section IV. Instead, the sampling frequency for SC-FDE in $\mathrm{MC}$ is set as the inverse of bit symbol duration $1 / T_{b}$ like the SC-FDE in conventional communication systems. In light of this, only a single sample is required within each symbol duration, which meets the low-complexity requirement for the nano-scale MC. In addition, the receiver starts to sample the concentration signal at the arrival time of its peak value in (7), which is proved to have the maximum signal-to-noise ratio (SNR) [6]. The length of ISI can be quantified by the metric in [50, eq. (31)], which neglects the taps with small channel gains compared with the largest one, defined as

$$
I=\underset{i}{\arg \max }\left\{\frac{h_{i}}{h_{0}} \geq \theta\right\},
$$

where $h_{i}=h\left(i T_{b}+t_{p}\right)$ is the channel gain of the $i$-th channel tap, and $\theta$ is the threshold for ISI length determination. Based on (5) and (8), (26) can be expanded as

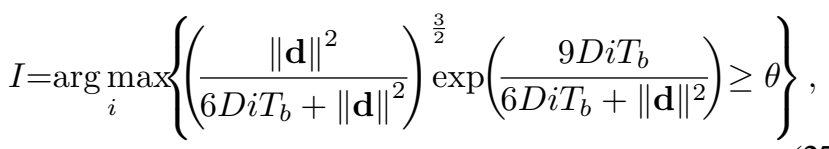

which shows the relationship between the length of ISI and the system parameters. Intuitively, the length of ISI $I$ is inversely proportional to the symbol duration $T_{b}$.

In this paper, $\mathrm{CP}$ acts as the GI to counteract the IBI and produce the circulant property for signal processing [51]. When its length is longer than that of the ISI, the IBI can be completely eliminated. After the removal of $\mathrm{CP}$, the sampled received signal at instant $t=m T_{b}+t_{p}$ has following expression as

$$
z_{m}=y_{m}+n_{m}=\sum_{i=0}^{L-1} h_{i} x_{(m-i) \bmod M}+n_{m},
$$

where $(\cdot) \bmod M$ represents the modulo- $M$ operation, $z_{m}=$ $z\left(m T_{b}+t_{p}\right), y_{m}=y\left(m T_{b}+t_{p}\right), x_{m-i}=x\left((m-i) T_{b}+t_{p}\right)$, and $n_{m}=n\left(m T_{b}+t_{p}\right)$, for $m=0,1, \ldots, M-1$. Additionally, the noisy received data block can be written in a vector form as

$$
\mathbf{z}=\left[z_{0}, \ldots, z_{m}, \ldots, z_{M-1}\right]^{\mathrm{T}}
$$

and it can expressed as

$$
\mathbf{z}=\mathbf{y}+\mathbf{n}=\mathbf{H} \mathbf{x}+\mathbf{n}
$$

where $\mathbf{y}=\left[y_{0}, \ldots, y_{m}, \ldots, y_{M-1}\right]^{\mathrm{T}}$ is the noiseless received data block, and we have

$$
\mathbf{x}=Q \mathbf{b}=\left[x_{0}, \ldots, x_{m}, \ldots, x_{M-1}\right]^{\mathrm{T}},
$$

as the transmit signal block according to (10) and (11). Based on (14), the noise block follows multivariate normal distribution, expressed as

$$
\mathbf{n}=\left[n_{0}, \ldots, n_{m}, \ldots, n_{M-1}\right]^{\mathrm{T}} \sim \mathcal{N}(\boldsymbol{\mu}, \boldsymbol{\Sigma})
$$

where $\boldsymbol{\mu}=[0, \ldots, 0, \ldots, 0]^{\mathrm{T}}$ is the $M$-dimensional mean vector, and $\boldsymbol{\Sigma}=\frac{1}{V_{\mathrm{R}}} \operatorname{diag}\left(\left[y_{0}, \ldots, y_{m}, \ldots, y_{M-1}\right]\right)$ is the $M \times M$ covariance matrix. Due to the $\mathrm{CP}$ insertion, $\mathbf{H}$ is an $M \times M$ circulant matrix, given by

$$
\mathbf{H}=\left(\begin{array}{cccccc}
h_{0} & 0 & \cdots & h_{L-1} & \cdots & h_{1} \\
h_{1} & h_{0} & 0 & & & \vdots \\
\vdots & \ddots & \ddots & \ddots & & \vdots \\
h_{L-1} & \cdots & h_{1} & h_{0} & 0 & 0 \\
\vdots & \ddots & & \ddots & h_{0} & 0 \\
0 & \cdots & h_{L-1} & \cdots & h_{1} & h_{0}
\end{array}\right)
$$

and its estimation is equivalent to estimating the CIR vector $\mathbf{h}=\left[h_{0}, \ldots, h_{i}, \ldots, h_{L-1}\right]^{\mathrm{T}}$ in the diffusion-based MC system.

The received data block in (29) is then converted into the frequency domain by multiplying an $M \times M$ DFT matrix $\mathbf{F}$, whose $(m, n)$ element $(0 \leq m, n \leq M-1)$ is $F_{m n}=$ $\sqrt{M} \exp \left(\frac{-j 2 \pi m n}{M}\right)$. Thus, the $M$-point DFT of the received signal is given by

$$
\overline{\mathbf{z}}=\mathbf{F} \mathbf{z}=\boldsymbol{\Lambda} \overline{\mathbf{x}}+\overline{\mathbf{n}}=\left[Z_{0}, \ldots, Z_{m}, \ldots, Z_{M-1}\right]^{\mathrm{T}},
$$

where $Z_{m}$ is the received signal at the $m$-th frequency. Due to the circulant property of $\mathbf{H}$, the channel matrix in the frequency domain has the following form

$$
\boldsymbol{\Lambda}=\mathbf{F H F}^{\mathrm{H}}=\operatorname{diag}\left(\left[H_{0}, \ldots, H_{m}, \ldots, H_{M-1}\right]\right),
$$

where $[\cdot]^{\mathrm{H}}$ represents conjugate transpose operation of the matrix, and $\boldsymbol{\Lambda}$ has a fixed form when channel matrix $\mathbf{H}$ stays constant. Besides, $\overline{\mathbf{x}}=\mathbf{F} \mathbf{x}=\left[X_{0}, \ldots, X_{m}, \ldots, X_{M-1}\right]^{\mathrm{T}}$ and $\overline{\mathbf{n}}=\mathbf{F n}=\left[N_{0}, \ldots, N_{m}, \ldots, N_{M-1}\right]^{\mathrm{T}}$ represent the transmit 


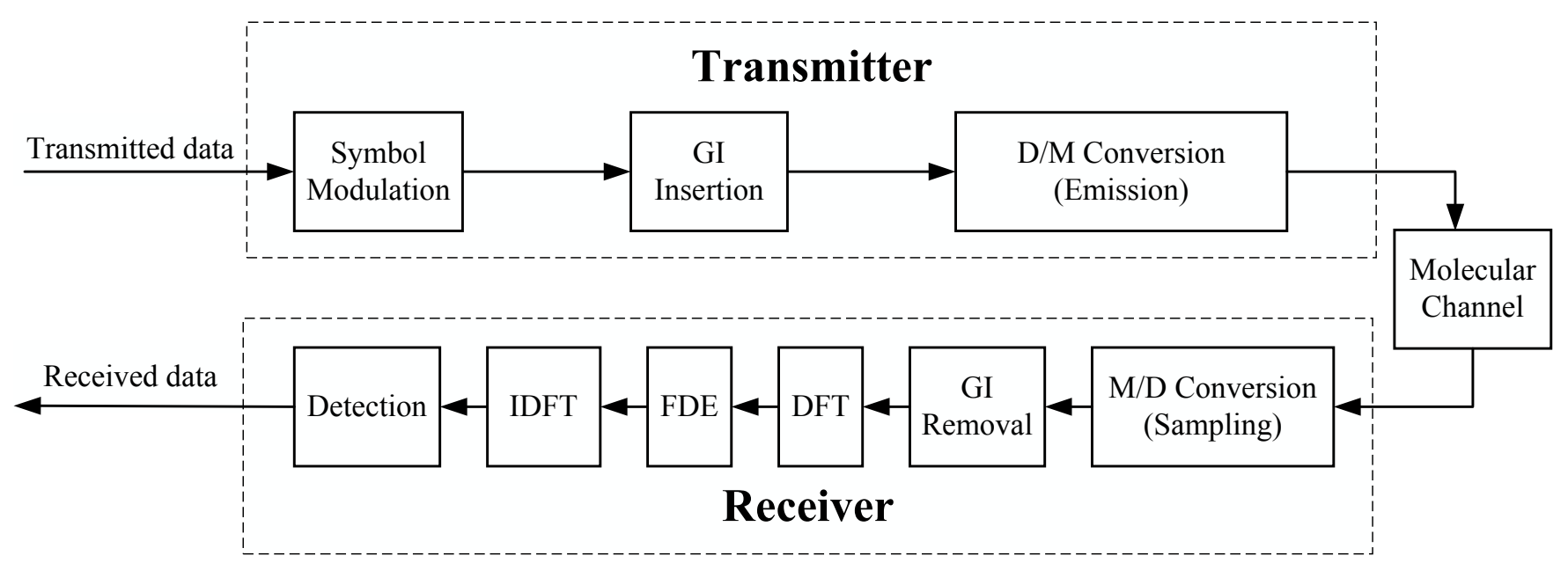

Fig. 3. Structure diagram of SC-FDE in molecular communication.

signal and noise vectors in the frequency domain, whose components at the $m$-th frequency are given by

$$
X_{m}=\frac{1}{\sqrt{M}} \sum_{n=0}^{M-1} x_{n} \exp \left(\frac{-j 2 \pi m n}{M}\right),
$$

and

$$
N_{m}=\frac{1}{\sqrt{M}} \sum_{n=0}^{M-1} n_{n} \exp \left(\frac{-j 2 \pi m n}{M}\right) .
$$

\section{Equalization Criteria}

In light of the demonstration in Fig. 3, the FDE is implemented after DFT to mitigate the ISI of the received data block. The equalized signal has the following form as

$$
\mathbf{W} \overline{\mathbf{z}}=\mathbf{W} \boldsymbol{\Lambda} \overline{\mathbf{x}}+\mathbf{W} \overline{\mathbf{n}},
$$

where $\mathbf{W}=\operatorname{diag}\left(\left[W_{0}, \ldots, W_{m}, \ldots, W_{M-1}\right]\right)$, is the linear equalization matrix at the receiver that is diagonal, and its weight $W_{m}$ at the $m$-th frequency tone depends on the equalization criterion.

1) ZF equalizer: Based on the analysis and discussion above, the nano-scale MC system has the constraint of limited computation capability. Considering the trade-off between error performance and complexity, the ZF equalizer is typically used to invert the channel and remove the ISI due to its easy implementation. The weight at the $m$-th frequency tone based on the $\mathrm{ZF}$ criterion is the reciprocal of the $m$-th main diagonal entry in $\Lambda$, given by

$$
W_{\mathrm{ZF}, m}=\frac{1}{H_{m}},
$$

and the corresponding equalization matrix $\mathbf{W}_{\mathrm{ZF}}$ is the inverse of $\Lambda$, given by

$$
\mathbf{W}_{\mathrm{ZF}}=\boldsymbol{\Lambda}^{-1}=\operatorname{diag}\left(\left[\frac{1}{H_{0}}, \ldots, \frac{1}{H_{m}}, \ldots, \frac{1}{H_{M-1}}\right]\right),
$$

where $(\cdot)^{-1}$ is the matrix inverse operation, and the derivation of $\mathbf{W}_{\mathrm{ZF}}$ requires sufficiently low computational complexity.
2) Optimal MMSE equalizer: Despite the easy implementation of the ZF equalizer, some noise components are enhanced at the frequency with low channel gains. To tackle this issue, the optimal MMSE equalizer is proposed, aiming at minimizing the mean square error, and its weight at the $m$-th frequency tone is given by

$$
\begin{aligned}
W_{\text {MMSE-opt }, m} & =\arg \min _{W_{m}} \mathbb{E}\left[\left|W_{m} Z_{m}-X_{m}\right|^{2}\right] \\
& =\frac{H_{m}^{*}}{\left|H_{m}\right|^{2}+\frac{\mathbb{E}\left[\left|N_{m}\right|^{2}\right]}{\mathbb{E}\left[\left|X_{m}\right|^{2}\right]}},
\end{aligned}
$$

where $\mathbb{E}\left[\left|X_{m}\right|^{2}\right]$ and $\mathbb{E}\left[\left|N_{m}\right|^{2}\right]$ are given by

$$
\mathbb{E}\left[X_{m}^{2}\right]=\frac{Q^{2}}{2}+\frac{Q^{2}}{4 M} \sum_{k=0}^{M-1} \sum_{l \neq k} \exp \left(\frac{j 2 \pi m(l-k)}{M}\right),
$$

and

$$
\mathbb{E}\left[N_{m}^{2}\right]=\frac{1}{M V_{\mathrm{R}}} \sum_{k=0}^{M-1} y_{k}
$$

respectively.

Proof: See Appendix E.

By substituting the results of (42) and (43) into (41), the weight at the $m$-th frequency tone in the optimal MMSE equalizer can be derived, and the corresponding equalization matrix has the form of $\mathbf{W}_{\mathrm{MMSE}-\text { opt }}=$ $\operatorname{diag}\left(\left[W_{\text {MMSE-opt }, 1}, \ldots, W_{\text {MMSE-opt }, m}, \ldots, W_{\text {MMSE-opt }, M}\right]\right)$.

Remark 4: A practical MC system cannot deploy the optimal MMSE equalizer as shown in (41). From (42), the signal power components in the frequency domain are calculable and frequency-dependent. On the contrary, the noise power components are independent of the frequency, which is unattainable in (43) as $y_{k}$ is the noiseless signal to be detected. The optimal MMSE equalizer in FDE is therefore signaldependent, and varied block by block. Yet, its TDE counterpart faces the same challenges [18]. Worse still, its weights are updated in a symbol-wise manner due to the presence of the signal-dependent counting noise, which is computationally cumbersome for the $\mathrm{MC}$ receiver. 
From the analysis above, the estimation of signal-dependent noise power becomes a critical issue in MC. To this end, two practical MMSE equalizers that has sub-optimal error performance are proposed from the statistical and iterative perspectives, respectively.

3) Statistical MMSE Equalizer: From Appendix E, the statistical information of the transmit signal is $b_{k} \sim \operatorname{Bernoulli}(p)$, and we assume $p=0.5$. Correspondingly, (43) can be averaged by this statistical information as

$$
\begin{aligned}
\hat{N}_{\mathrm{ST}}^{2} & =\frac{Q}{M V_{\mathrm{R}}} \sum_{k=0}^{M-1} \mathbb{E}_{b_{k-l}}\left[\sum_{l=0}^{M-1} h_{l} b_{(k-l) \bmod M}\right] \\
& =\frac{Q}{2 V_{\mathrm{R}}} \sum_{l=0}^{M-1} h_{l},
\end{aligned}
$$

and it is a deterministic value, which can be calculated prior to the equalization process and be applied to all the blocks regardless of the block variance. Thus, $\hat{N}_{\mathrm{ST}}^{2}$ can replace the unattainable component $\mathbb{E}\left[N_{m}^{2}\right]$ in (43), attaining the new equalization matrix $\mathbf{W}_{\text {MMSE-stat }}$ instead of $\mathbf{W}_{\text {MMSE-opt }}$. Consequently, it is referred to as the statistical MMSE equalizer.

4) Iterative MMSE Equalizer: Alternatively, taking the signal-dependent characteristics of the noise into account, the noise power components vary block-by-block. Instead of the statistical estimation, the DF of the detected symbols can facilitate the noise power estimation in each block, given by

$$
\hat{N}_{\mathrm{EQ}}^{2}=\frac{1}{M V_{\mathrm{R}}} \sum_{k=0}^{M-1} y_{\mathrm{EQ}, k},
$$

where $y_{\mathrm{EQ}, k}$ is the recovery of the noiseless received signal sampled at the $k$-th symbol duration based on the practical equalizers, serving as the DF.

Note that $\hat{N}_{\mathrm{EQ}}^{2}$ is attainable that can substitute $\mathbb{E}\left[N_{m}^{2}\right]$ in (41) to obtain the practical equalization matrix with the MMSE criterion. In particular, the noise estimation of (45) can be achieved via an iterative manner, and the input is required at the beginning from the detection results of some practical equalizers, such as the linear ZF equalizer or the statistical MMSE equalizer. After that, its output can be either the equalization result or the input for the next iteration. Against this background, this DF-aided scheme is coined as the iterative MMSE equalizer, and the corresponding equalization matrix is denoted as $\mathbf{W}_{\text {MMSE-iter }}$.

\section{Signal Detection}

After the FDE process, IDFT matrix $\mathbf{F}^{\mathrm{H}}$ multiplies both sides of (38), transforming the received data block back to the time domain as

$$
\hat{\mathbf{z}}=\mathbf{F}^{\mathrm{H}} \mathbf{W} \overline{\mathbf{z}}=\mathbf{F}^{\mathrm{H}} \mathbf{W} \boldsymbol{\Lambda} \overline{\mathbf{x}}+\mathbf{F}^{\mathrm{H}} \mathbf{W} \overline{\mathbf{n}}
$$

When the ZF equalizer is considered, i.e., $\mathbf{W}=\mathbf{W}_{\mathrm{ZF}}$, the $M$-length detected data block after IDFT is given by

$$
\begin{aligned}
\hat{\mathbf{z}}_{\mathrm{ZF}} & =\mathbf{F}^{\mathrm{H}} \overline{\mathbf{x}}+\mathbf{F}^{\mathrm{H}} \mathbf{W}_{\mathrm{ZF}} \overline{\mathbf{n}} \\
& =\mathbf{F}^{\mathrm{H}} \mathbf{F} \mathbf{x}+\mathbf{F}^{\mathrm{H}} \mathbf{F} \mathbf{H}^{-1} \mathbf{F}^{\mathrm{H}} \mathbf{F} \mathbf{n} \\
& =\mathbf{x}+\mathbf{H}^{-1} \mathbf{n},
\end{aligned}
$$

TABLE I

COMPUTATIONAL COMPLEXITY COMPARISON BETWEEN DIFFERENT RECEIVERS IN EACH DATA BLOCK

\begin{tabular}{ll}
\hline Receiver Type & Computational complexity \\
\hline MMSE-TDE [18] & $\mathcal{O}\left(M\left(L^{3}+2 L^{2}+L\right)\right)$ \\
MLSD with Viterbi algorithm [18] & $\mathcal{O}\left(M L 2^{L}\right)$ \\
ZF-FDE & $\mathcal{O}\left(2 M \log _{2} M+M\right)$ \\
Statistical MMSE-FDE & $\mathcal{O}\left(2 M \log _{2} M+M\right)$ \\
$i$-times Iterative MMSE-FDE & $\mathcal{O}\left((i+2) M \log _{2} M+(i+1) M\right)$ \\
\hline
\end{tabular}

where $\mathbf{H}^{-1} \mathbf{n} \sim \mathcal{N}\left(\mathbf{H}^{-1} \boldsymbol{\mu}, \mathbf{H}^{-1} \boldsymbol{\Sigma}\left(\mathbf{H}^{-1}\right)^{\mathrm{H}}\right)$ according to (32), and its elements also follow normal distribution with zero mean. Similarly, the $M$-length detected data block with the MMSE equalization after IDFT is given by

$$
\begin{aligned}
\hat{\mathbf{z}}_{\mathrm{MMSE}} & =\mathbf{F}^{\mathrm{H}} \mathbf{W}_{\text {MMSE }} \boldsymbol{\Lambda} \overline{\mathbf{x}}+\mathbf{F}^{\mathrm{H}} \mathbf{W}_{\text {MMSE }} \overline{\mathbf{n}} \\
& =\mathbf{F}^{\mathrm{H}} \mathbf{W}_{\text {MMSE }} \mathbf{F H} \mathbf{F}^{\mathrm{H}} \mathbf{F} \mathbf{x}+\mathbf{F}^{\mathrm{H}} \mathbf{W}_{\text {MMSE }} \mathbf{F n} \\
& =\boldsymbol{\Gamma} \mathbf{H} \mathbf{x}+\boldsymbol{\Gamma} \mathbf{n},
\end{aligned}
$$

where $\boldsymbol{\Gamma}=\mathbf{F}^{\mathrm{H}} \mathbf{W}_{\text {MMSE }} \mathbf{F}$ is a ciruculant matrix, and $\boldsymbol{\Gamma n} \sim$ $\mathcal{C N}\left(\boldsymbol{\Gamma} \boldsymbol{\mu}, \boldsymbol{\Gamma} \Sigma \boldsymbol{\Gamma}^{\mathrm{H}}\right)$ is the noise term, indicating that the noise components also obey normal distribution with zero mean. In particular, this equalization process is applicable to both statistical and iterative MMSE equalizers.

Denote $\hat{\mathbf{z}}=\left[\hat{z}_{0}, \ldots, \hat{z}_{m}, \ldots, \hat{z}_{M-1}\right]^{\mathrm{T}}$, which determines the recovery of the binary data block, as $\hat{\mathbf{b}}=$ $\left[\hat{b}_{0}, \ldots, \hat{b}_{m}, \ldots, \hat{b}_{M-1}\right]^{\mathrm{T}}$. From the previous analysis, the threshold based detection of the $m$-th bit, $\hat{b}_{m}$, is given by

$$
\hat{b}_{m}= \begin{cases}1, & \text { when } \hat{z}_{m} \geq \frac{Q}{2}, \\ 0, & \text { otherwise. }\end{cases}
$$

\section{E. Comparison of Computational Complexity}

The computational complexity is measured in terms of the real/complex multiplication numbers in each data block [52], and the comparison between the proposed FDEs and timedomain detectors, i.e., TDE with MMSE criterion and the optimal maximum likelihood sequence detection (MLSD) is shown in Table $\mathrm{I}^{1}$, where $M$ and $L$ refer to the symbol length in each block and the number of channel taps, respectively.

Intuitively, the computational complexity of the all the schemes is proportional to the symbol length $M$. The equalization process of (38) in FDEs only accounts for $\mathcal{O}(M)$ complexity order due to the sparse nature of the diagonal matrix. In addition to this, FDE requires both DFT and IDFT for the frequency/time domain transform with complexity order of $\mathcal{O}\left(M \log _{2} M\right)$, respectively. Despite the immunity to ISI, FDEs are more sensitive to $M$ but only with a logarithmic growth. For the iterative MMSE-FDE with $i$-times iterations for the noise power estimation, the extra $i$-times FDE and IDFT operations are required. Nevertheless, the time-domain signal processing approaches display third-order polynomial or even exponential growth with $L$, which is computationally prohibitive for the high symbol rate case.

\footnotetext{
${ }^{1}$ The equalization matrix for ZF-FDE and statistical MMSE-FDE is static if the CIR is fixed. The computational complexity of this process is thus neglected.
} 


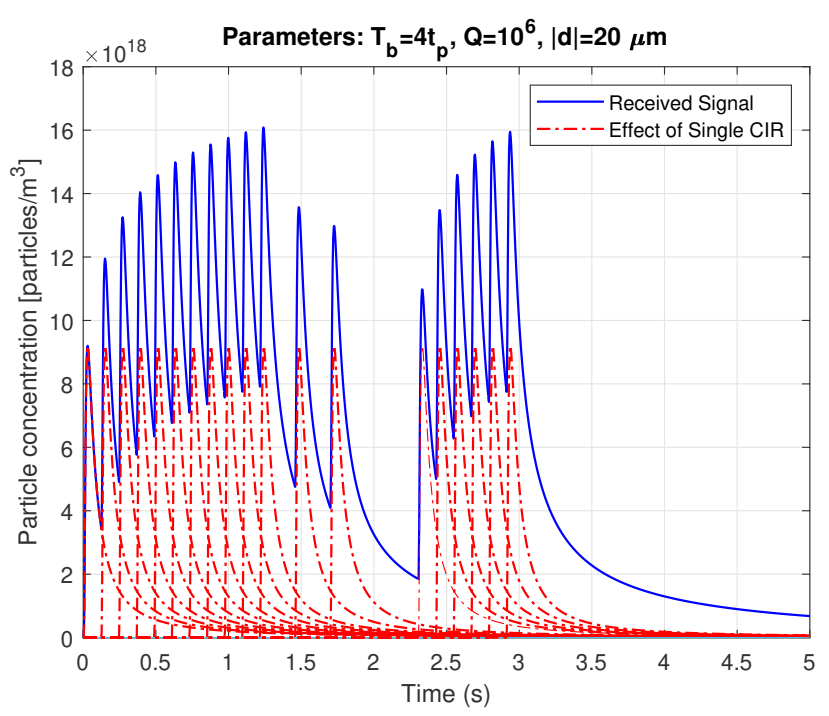

Fig. 4. Consecutive transmission when $T_{b}=4 t_{p}$.

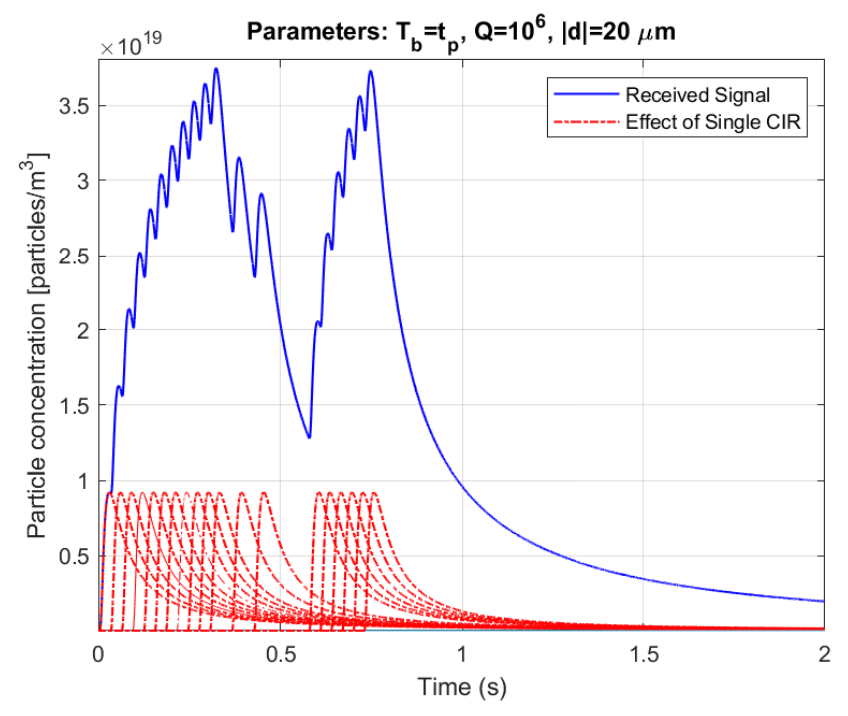

Fig. 5. Consecutive transmission when $T_{b}=t_{p}$.

In particular, the time-domain MC techniques deal with the real and positive concentration signal due to its nature. Hence, real multiplication is applied in the time-domain $\mathrm{MC}$, while complex multiplication in the frequency-domain MC.

\section{Numerical RESUlts}

This section presents the simulation results for the received signal in response to the consecutive transmission, the frequency response of the free diffusion MC channel, the recovery performance of sampling frequency, and the bit error rate (BER) of SC-FDE for MC with ZF and MMSE criteria. We have $r=0.1 \mu \mathrm{m}, D=2.2 \times 10^{-9} \mathrm{~m}^{2} / \mathrm{s}$, and $\theta=0.01$ throughout the simulation part for the counting noise model and other essential parameters are either displayed in the figure caption or stated in the corresponding context.

The symbol duration significantly impacts signal detection performance in a communication system, and MC is no exception. For an intuitive exposition, we exemplify the noiseless

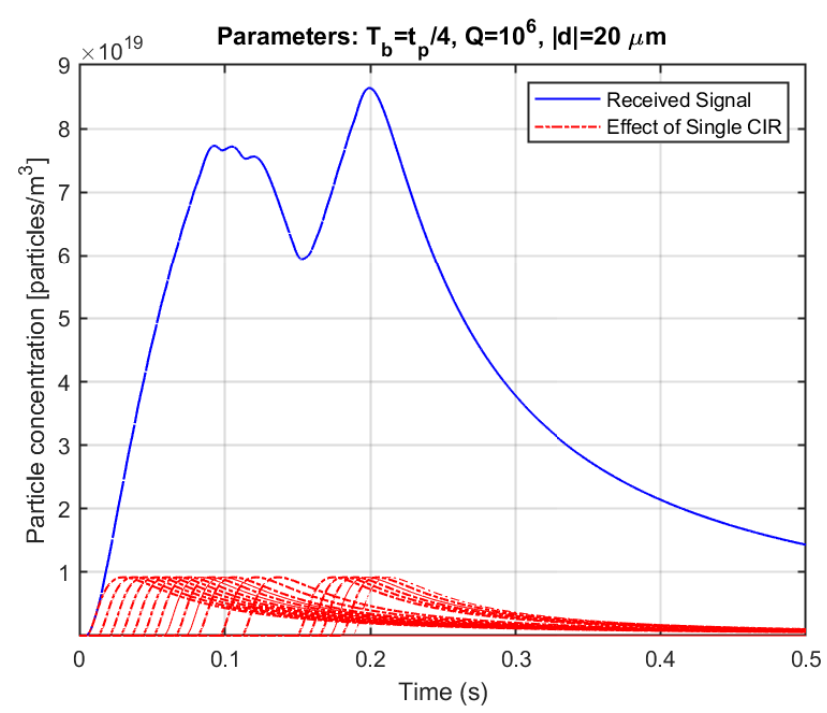

Fig. 6. Consecutive transmission when $T_{b}=\frac{t_{p}}{4}$.

received signals of a consecutive transmission of the data block $[1,1,1,1,1,1,1,1,1,1,1,0,1,0,1,0,0,0,0,1,1,1,1,1,1]$. Various settings of symbol duration with respect to the arrival time of peak concentration in (7) are considered as $T_{b}=\left[4 t_{p}, t_{p}, \frac{t_{p}}{4}\right]$ in Fig.s 4, 5, and 6, respectively, which correspond to conventional transmission, high symbol rate transmission, and ultra high symbol rate transmission.

Notably, when bit-1 is transmitted, the effect of single CIR (red line with dots) is imposed on the receiver side, while there is no impact of the bit- 0 transmission. The receiver senses the superposition of these CIRs, namely, the received signal (blue line), with which the signal detection can be implemented. Note that the transmission rate (symbol duration) and the error performance (ISI effect) are the fundamental trade-off in the MC system. Figure 4 shows the received signal with the longest symbol duration among these figures as $T_{b}=4 t_{p}$. The received signal captures the characteristics of the binary data block based on OOK modulation. Hence, the information can be easily recovered via conventional fixed or adaptive threshold based detection methods. The symbol duration in Fig. 5 is the same as the arrival time of peak concentration value, i.e., $T_{b}=t_{p}$, and the binary data block feature in Fig. 5 cannot be distinctly manifested from the received signal, as in Fig. 4. Nevertheless, the received signal in Fig. 5 demonstrates the rising edge feature of bit- 1 transmission, and some differential detection schemes may be able to recover the original information in this case [53], [54].

In Fig. 6, the feature of the transmitted binary data block is overwhelmed by the severe ISI influence due to the relatively short symbol duration compared to traditional MC system, namely, $T_{b}=\frac{t_{p}}{4}$. In this case, some conventional time-domain signal processing techniques are incapable of dealing with such a high data symbol transmission due to either the loss of detection metrics (e.g., inflexion feature) [55], [56], or the prohibitively high computational complexity [18]. The reasons above motivate us to design the FDE for MC in favor of its lower computational complexity. 

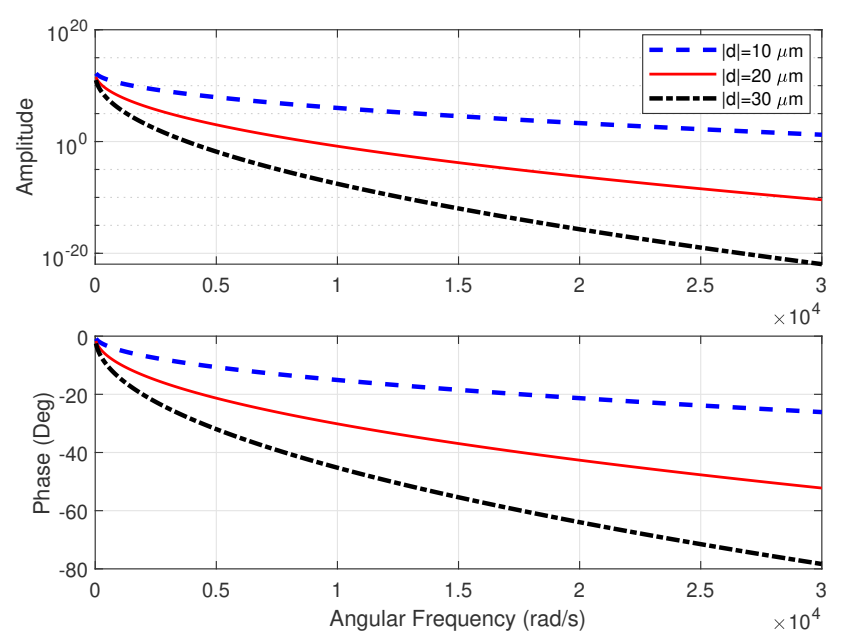

Fig. 7. Amplitude and phase of frequency response with respect to the transmission distance.

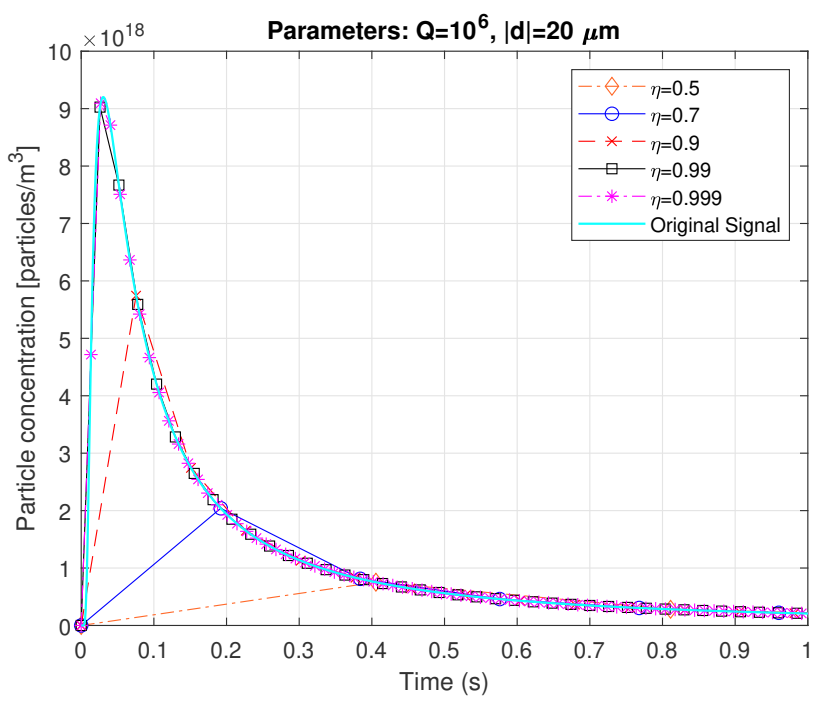

Fig. 8. One-shot concentration signal with different sampling frequencies with regard to the $\eta$ values.

The frequency response is shown in Fig. 7. We observe that the amplitude of the frequency response, given by (17), monotonically decreases with the increasing angular frequency. Hence, the free diffusion MC channel has the low-pass feature in the frequency domain. The phase component follows a similar trend as the amplitude component does along the $\mathrm{x}$-axis, decaying with the increase of angular frequency. Furthermore, Fig. 7 depicts the effect of transmission distance on frequency response. We find that the longer the transmission distance, the more rapid decline of both amplitude and phase components along the angular frequency, which is reasonable as the increase of transmission distance leads to higher pathloss.

Figure 8 considers the one-shot transmission, and the relationship between sampling frequency and the energy portion $\eta$ that first appears in (22), is revealed. The sampling frequency

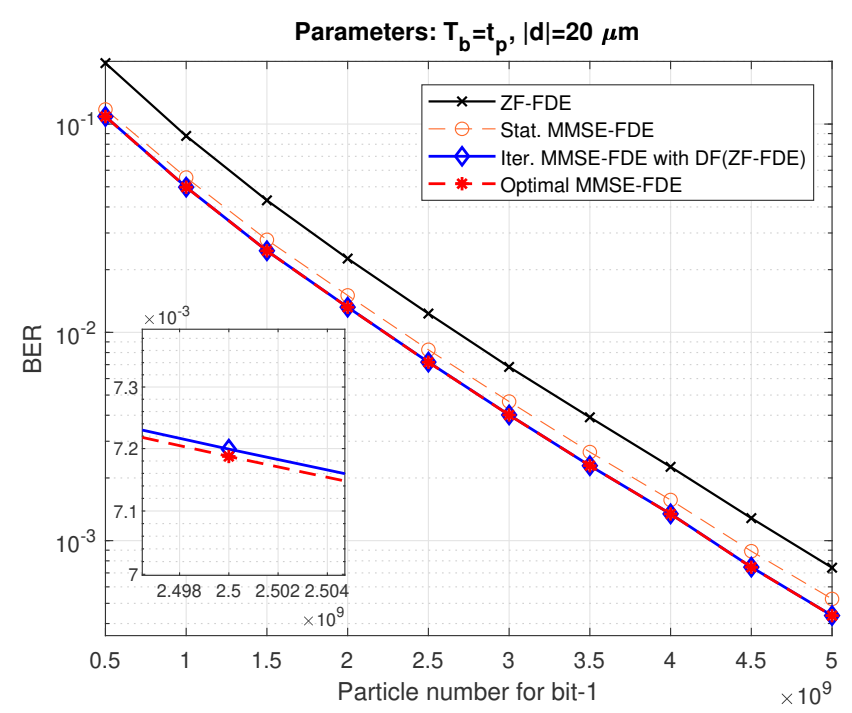

Fig. 9. BER performance with different types of equalizers with molecule number for bit-1 (perfect CSI).

in Fig. 8 is $F_{s}=\frac{\omega_{c}}{\pi}$, and the expression of $\omega_{c}$ is derived in (23). The original signal is set as the benchmark to compare with the simulation results with different $\eta$ values, and it is obtained via an extremely high sampling frequency, which may not be available in practice. Explicitly, the greater $\eta$, the better signal recovery. It can be found that when $\eta=[0.5,0.7,0.9]$, the signal cannot be well recovered since the peak value of the original signal is not sampled due to the low sampling frequency, which may cause the estimation error of channel and distance. The signal can be almost regenerated when the peak value is sampled in the scenarios of $\eta=0.99$ and $\eta=0.999$. However, such large $\eta$ values indicate a fast sampling frequency, which is a challenging task for the nanoscale receiver due to its size constraint. Thus, the trade-off between sampling frequency and the recovery performance should be considered in the MC system. Furthermore, such sampling frequency is proposed for one-shot transmission instead of the consecutive transmission.

Figure 9 shows the BER performance of the SC-FDE with various types of equalizers, where the perfect CSI is assumed. Particularly, the optimal MMSE equalizer is treated as a benchmark for the evaluation of other MMSE equalizers. The iterative MMSE equalizers adopt the DF from the linear ZF equalizer as the input for noise power estimation. Keeping the receiver scale of MC in mind, only the first-time iteration result is used as DF. Different particle numbers for bit-1 transmission are considered in Fig. 9, and the increasing number of molecules for bit-1 transmission can contribute to the BER performance enhancement. When the counting noise model is considered, the time-domain SNR with OOK modulation is proportional to the number of molecules for the bit-1 transmission [6]. The transmission rate is $T_{b}=t_{p}$, and the length of ISI corresponds to $I=57$ based on the previously defined metric. To prevent the impact of IBI, the $\mathrm{CP}$ has the same length as ISI; the original transmission symbol in each data block is 4 times the size of the CP, i.e., $M=228$. The optimal MMSE-FDE has the best performance, 


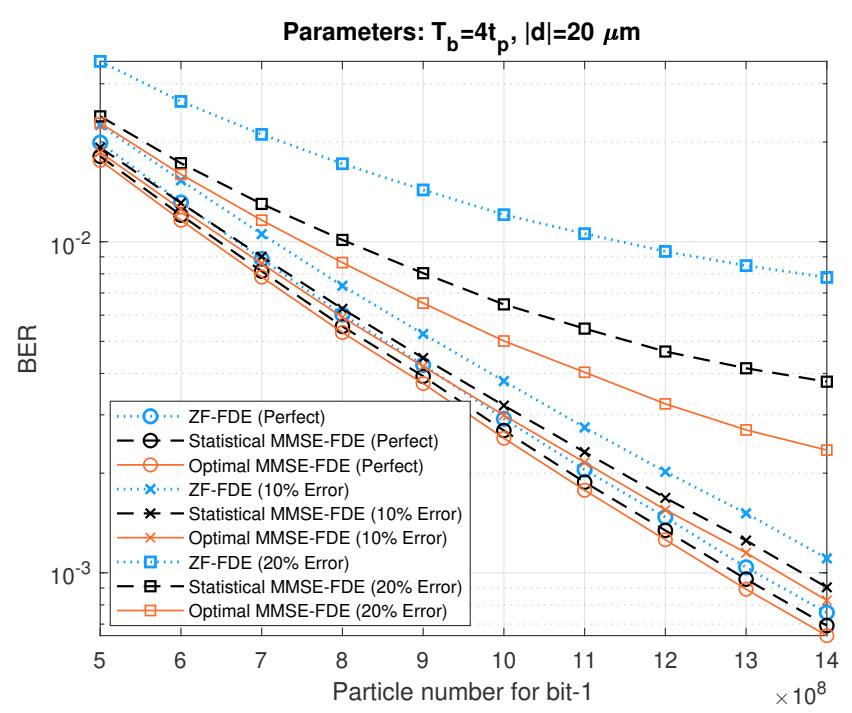

Fig. 10. BER performance of SC-FDE with different levels of channel estimation error.

and all the MMSE-FDE equalizers outperform the linear ZF-FDE equalizer as they can avoid the noise amplification phenomenon. The statistical MMSE-FDE that solely depends on the statistical information of the transmit signal has the worst BER performance among the MMSE-FDE schemes. The iterative MMSE-FDE with DF from ZF-FDE is slightly inferior to the optimal MMSE-FDE, while they almost share the same performance in the low to high SNR regimes.

The robustness of the conventional detection schemes concerning the channel estimation error has been studied in [57], where the error is defined as the zero-mean Gaussian noise with the variance related to the original channel gains. Following this metric, Fig. 10 shows the error performance of SC-FDE concerning the channel estimation error, where the linear ZF-FDE, statistical MMSE-FDE, and optimal MMSEFDE are considered. Note that the iterative MMSE-FDE is omitted here since it exhibits a similar performance to the optimal MMSE-FDE. Here, the transmission rate is $T_{b}=4 t_{p}$, and the length of ISI corresponds to 15 . Again, in the same CSI error condition, the optimal MMSE-FDE demonstrates the best BER performance, and the ZF-FDE has the worst. Besides, Fig. 10 shows that the more accurate the CSI at the receiver side, the better the BER performance of the FDEs. Hence, all the FDEs with perfect CSI exhibit better performance than their counterparts with imperfect CSI. The FDEs with $10 \%$ CSI error are inferior to those with perfect CSI, and the FDEs with $20 \%$ CSI error suffer from the worst BER performance.

Figures 11 and 12 compare the BER performance between our proposed FDEs and the conventional time-domain detection techniques in [18]. A high transmission rate is considered in Fig. 11, where $T_{b}=\frac{t_{p}}{2}$, and the corresponding length of ISI is 114. In this case, the implementation cost of MLSD is prohibitively high, even with complexity reduction from the Viterbi algorithm. Hence, the BER performance of MLSD is omitted in Fig. 11. Provided with a long length of ISI, an extended length of CP is required in SC-FDE, which deteriorates the throughput efficiency. The channel shortening equalizers

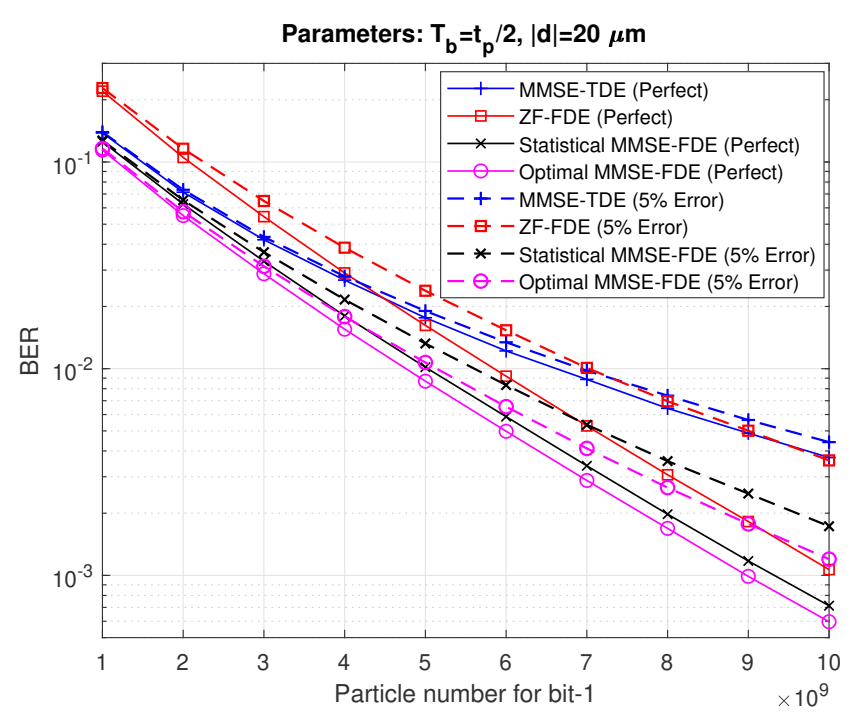

Fig. 11. BER performance of TDE and FDEs (high transmission rate scenario).

developed in conventional wired and wireless communication systems can be deployed to obtain the target impulse response with a shorter number of channel taps [58], [59]. Consequently, the required length of $\mathrm{CP}$ can be reduced. To the best of our knowledge, due to the channel model difference between the conventional communication systems and $\mathrm{MC}$, the proposed channel shortening equalizers cannot be applied to MC directly, and further independent research should be conducted. Additionally, a non-linear mapping technique at the receiver side is also envisaged to shorten the number of channel taps in MC according to its signal characteristics [60].

From the results demonstrated in Fig. 11, the optimal MMSE-FDE again exhibits the best BER performance in the simulated SNR region, but it has a slight advantage over the statistical MMSE-FDE. The TDE and FDEs with MMSE criteria have similar BER performance in the low SNR regime, where the MMSE-TDE is slightly inferior to the MMSEFDEs. The ZF-FDE shows the worst performance among these equalization methods. With the increasing SNR, the gap between the TDE and FDEs becomes more extensive, and ZF-FDE surpasses the MMSE-TDE when the SNR value is moderate. Though the TDE and FDEs endure the same length of ISI in the simulation of Fig. 11, CP is added in FDEs, which deteriorates their throughput efficiency in practice. In light of this, the FDEs with lower computational complexity can outperform their TDE counterpart with higher computational complexity. However, when the CSI error is $5 \%$ under the configuration of Fig. 11, FDEs are more sensitive to the channel estimation error than the MMSE-TDE.

On the contrary, Fig. 12 considers the scenario with relatively slow transmission rates, where $T_{b}=8 t_{p}$ and $T_{b}=16 t_{p}$. The corresponding lengths of ISI are 8 and 4, respectively. Hence, the MLSD can be easily obtained. When the transmission rate is fixed, MLSD has the best BER performance with a significant advantage over other detection schemes. The MMSE-TDE and the optimal MMSE-FDE show the comparable BER performance when $T_{b}=8 t_{p}$. Alternatively, the 


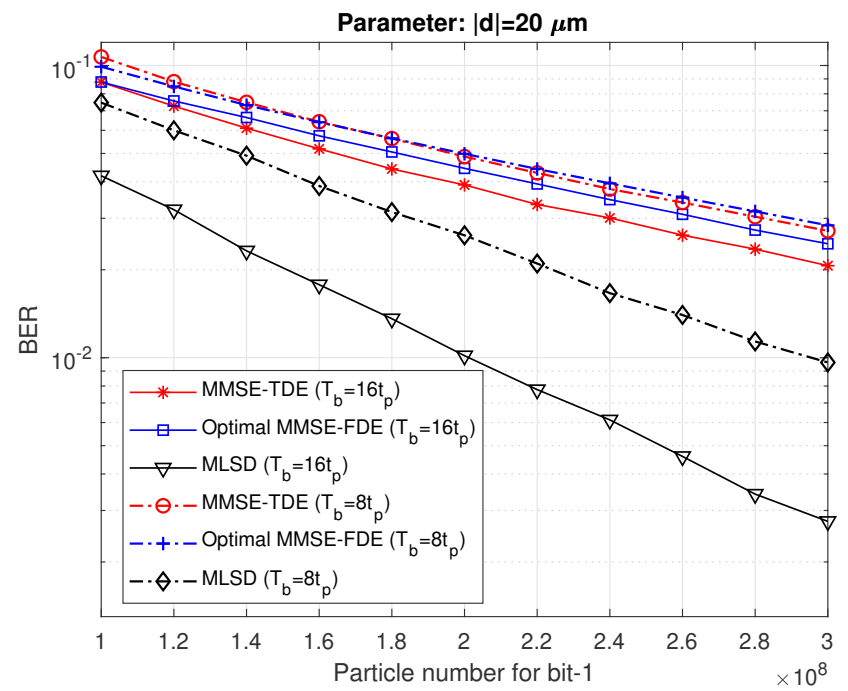

Fig. 12. BER performance of TDE, FDE, and MLSD (low transmission rate scenario).

MMSE-TDE is slightly superior to the optimal MMSE-FDE in terms of BER performance when the transmission rate falls to $T_{b}=16 t_{p}$. The MMSE-TDE has higher computational complexity according to Table I, while the FDE deploys the CP to prevent IBI, which inevitably decreases the throughput efficiency. The advantage of MMSE-TDE emerges when the ISI effect is alleviated due to the decrease of the transmission rate. Furthermore, under the same molecular energy, i.e., released particle number for bit-1, the transmission rate reduction leads to enhanced BER performance. Intuitively, this is due to the suppression of the ISI effect.

From the numerical results above, we can find that the low-complexity FDEs can obtain desirable BER performance compared with their time-domain counterparts, especially for the scenario with high transmission rates. For achieving the same BER performance, MMSE-FDEs require fewer transmitted particles than the linear ZF-FDE. However, MMSE equalizers' performance gain is obtained at the cost of higher computational complexity, especially when the iteration is taken into account.

\section{CONCLUSION AND FUTURE WORKS}

While MC has been thoroughly researched in the time domain, its frequency-domain analysis brings new insights. For the first time, this paper studied the frequency response for the diffusion-based MC system, which intuitively shows the lowpass characteristics. The CIR was proved to have finite energy, and the definitions of bandwidth and sampling frequency were proposed for the one-shot transmission signal. Interestingly, the FDE in MC was found to be the SC-FDE in nature, which overcomes the drawbacks of the TDE in MC, realizing the high symbol rate transmission with a desirable trade-off between the error performance and computational complexity. The SC-FDE with MMSE criterion in the diffusion-based $\mathrm{MC}$ is different from the wireless communication due to the different characteristics of the noise model. In light of this, the sub-optimal MMSE equalizers are proposed with the counting noise power estimation schemes, achieving almost the same BER performance with the optimal MMSE equalizer.

Provided with the frequency response of the MC channels, the signal processing no longer relies on the time-domain analysis only, but has its extension to the frequency domain. Consequently, the advanced FDE, channel estimation, channel shortening, pulse shaping, and other related aspects in $\mathrm{MC}$ can be researched from the frequency-domain perspective.

\section{APPENDIX A PROOF OF PROPOSITION 1}

In the free diffusion channel, the integration of $|h(t)|$ over the whole time region is given by

$$
\begin{aligned}
\int_{-\infty}^{\infty}|h(t)| d t & =\int_{0}^{\infty} h(t) d t \\
& =\gamma \int_{0}^{\infty} \exp \left(-\frac{\alpha}{t}\right)\left(\frac{1}{t}\right)^{\frac{3}{2}} d t \\
& =\gamma \sqrt{\frac{\pi}{\alpha}} \operatorname{erf}(\infty) .
\end{aligned}
$$

Given $\lim _{x \rightarrow \infty} \operatorname{erf}(x)=1$ and (9), (50) can be re-written as

$$
\int_{-\infty}^{\infty}|h(t)| d t=\frac{1}{4 \pi\|\mathbf{d}\| D}
$$

In light of (51), when both $\|\mathbf{d}\|$ and $D$ are finite, the integration of CIR over time axis converges to a finite value $\frac{1}{4 \pi\|\mathbf{d}\| D}$ as time approaches to the infinity. In this scenario, $h(t)$ is proved to be an absolutely integrable function over the time axis.

\section{APPENDIX B \\ FREQUENCY RESPONSE OF CIR}

The frequency response of CIR is the Fourier transform of (8), given by

$$
H(\omega)=\int_{-\infty}^{\infty} h(t) \exp (-j \omega t) d t .
$$

For ease of exposition, let $\beta=j \omega$, and (52) can be expanded as

$$
\begin{aligned}
H(\omega)= & \gamma \int_{0}^{\infty} \frac{\exp \left(-\frac{\alpha}{t}-\beta t\right)}{t^{\frac{3}{2}}} d t \\
= & \gamma \int_{0}^{1} \frac{\exp \left(-\sqrt{\alpha \beta}\left(\frac{1}{\sqrt{\frac{\beta}{\alpha}} t}+\sqrt{\frac{\beta}{\alpha}} t\right)\right)}{t^{\frac{3}{2}}} d t \\
& +\gamma \int_{1}^{\infty} \frac{\exp \left(-\sqrt{\alpha \beta}\left(\frac{1}{\sqrt{\frac{\beta}{\alpha}} t}+\sqrt{\frac{\beta}{\alpha}} t\right)\right)}{t^{\frac{3}{2}}} d t .
\end{aligned}
$$

Then, by defining $e^{-u}=\sqrt{\frac{\beta}{\alpha}} t$ when $t$ is in the interval of $[0,1)$, and $e^{u}=\sqrt{\frac{\beta}{\alpha}} t$ when $t$ lies in the interval of $[1, \infty)$, 
(53) can be re-expressed as

$$
\begin{aligned}
H(\omega)= & \gamma \sqrt[4]{\frac{\beta}{\alpha}} \int_{0}^{\infty} \exp \left(-\sqrt{\alpha \beta}\left(e^{u}+e^{-u}\right)\right) e^{\frac{u}{2}} d u \\
& +\gamma \sqrt[4]{\frac{\beta}{\alpha}} \int_{0}^{\infty} \exp \left(-\sqrt{\alpha \beta}\left(e^{-u}+e^{u}\right)\right) e^{-\frac{u}{2}} d u \\
= & 2 \gamma \sqrt[4]{\frac{\beta}{\alpha}} \int_{0}^{\infty} \exp \left(-2 \sqrt{\alpha \beta}\left(\frac{e^{-u}+e^{u}}{2}\right)\right) \frac{e^{\frac{u}{2}}+e^{-\frac{u}{2}}}{2} d u \\
= & 2 \gamma \sqrt[4]{\frac{\beta}{\alpha}} \int_{0}^{\infty} \exp (-2 \sqrt{\alpha \beta} \cosh (u)) \cosh \left(\frac{u}{2}\right) d u \\
= & 2 \gamma \sqrt[4]{\frac{\beta}{\alpha}} K_{\frac{1}{2}}(2 \sqrt{\alpha \beta})
\end{aligned}
$$

where $K_{m}(x)$ is the modified Bessel function of the second kind (Macdonald function) [61, Eq. (10.32.9)], given by

$$
K_{m}(z)=\int_{0}^{\infty} \exp (-z \cosh (x)) \cosh (m x) d x
$$

for $\angle z<\frac{\pi}{2}$. The modified spherical Bessel function $k_{m}(z)$ of order $m$ and argument $z$ has an explicit form given by [61, Eq. (10.49.12)]. Hence, it can replace the modified Bessel function given in (55). The result of [61, Eq. (10.47.9)] shows the relationship between the modified spherical Bessel function and the modified Bessel function as

$$
\begin{aligned}
k_{m}(z) & =\sqrt{\frac{\pi}{2 z}} K_{ \pm\left(m+\frac{1}{2}\right)}(z) \\
& =\frac{\pi}{2} \exp (-z) \sum_{k=0}^{m} \frac{a_{k}\left(m+\frac{1}{2}\right)}{z^{k+1}},
\end{aligned}
$$

where we have

$$
a_{k}\left(m+\frac{1}{2}\right)= \begin{cases}\frac{(m+k) !}{2^{k} k !(m-k) !}, & k=0,1, \ldots, m \\ 0, & k=m+1, m+2, \ldots\end{cases}
$$

By substituting (57) into (56), we can obtain $k_{0}(z)=$ $\frac{\pi}{2 z} \exp (-z)$. In light of this, the frequency response has a closed-form expression below

$$
\begin{aligned}
H(\omega) & =4 \gamma \sqrt{\frac{\beta}{\pi}} k_{0}(2 \sqrt{\alpha \beta}) \\
& =\frac{1}{4 \pi\|\mathbf{d}\| D} \exp \left(-\|\mathbf{d}\| \sqrt{\frac{j \omega}{D}}\right) .
\end{aligned}
$$

\section{APPENDIX C}

\section{SIGNAL ENERGY IN THE FREQUENCY DOMAIN}

For the free diffusion channel, we have the frequencydomain energy in the single side band as

$$
\frac{1}{\pi} \int_{0}^{\infty}|H(\omega)|^{2} d \omega=\frac{\gamma^{2}}{\alpha} \int_{0}^{\infty} \exp (-2 \sqrt{2 \alpha \omega}) d \omega
$$

Letting $u=2 \sqrt{2 \alpha \omega}$, (59) can be re-written as

$$
\begin{aligned}
\frac{1}{\pi} \int_{0}^{\infty}|H(\omega)|^{2} & =\frac{\gamma^{2}}{4 \alpha^{2}} \int_{0}^{\infty} u \exp (-u) d u \\
& =\frac{1}{16 \pi^{3}\|\mathbf{d}\|^{4} D} .
\end{aligned}
$$

\section{APPENDIX D}

\section{BANDWIDTH OF FREE DIFFUSION CHANNEL}

Based on the integrals in (60) of Appendix C, (22) can be rearranged as

$$
\frac{2 \sqrt{2 \alpha \omega_{c}}+1}{\exp \left(2 \sqrt{2 \alpha \omega_{c}}\right)}=1-\eta .
$$

By multiplying $-\exp (-1)$ on both sides of $(61)$, it has the following form, i.e.,

$$
\frac{-2 \sqrt{2 \alpha \omega_{c}}-1}{\exp \left(2 \sqrt{2 \alpha \omega_{c}}+1\right)}=\frac{\eta-1}{\exp (1)} .
$$

Define $x=-2 \sqrt{2 \alpha \omega_{c}}-1$ and $z=\frac{\eta-1}{\exp (1)}$, and thus, (62) can be converted to the following form as

$$
x \exp (x)=z .
$$

Specifically, the solution for $x$ in (63) is the Lambert W function $W_{k}(z)$, in which the subscript $k$ indicates the $k$-th branch of the Lambert W function [39]. Note that in (63), $z$ is only valid over the interval $[-\exp (-1), \infty)$. When $z$ has a real value, and it is in the interval $[-\exp (-1), 0)$, then $x$ has two possible values, i.e., $W_{0}(z) \in[-1,0)$ or $W_{-1}(z) \in$ $(-\infty,-1]$. Alternatively, if $z$ is in the interval $[0, \infty)$, then $x$ has a single result as $W_{0}(z)$.

Here, $x=-2 \sqrt{2 \alpha \omega_{c}}-1<-1$ and $-\exp (-1)<z=$ $\frac{\eta-1}{\exp (1)}<0$. Against this background, the solution for $x$ in (63) is

$$
x=-2 \sqrt{2 \alpha \omega_{c}}-1=W_{-1}\left(\frac{\eta-1}{\exp (1)}\right) .
$$

Based on (9), the corresponding bandwidth with respect to the proportion value $\eta$ has the form of

$$
\omega_{c}=\frac{D\left(W_{-1}\left(\frac{\eta-1}{\exp (1)}\right)+1\right)^{2}}{2\|\mathbf{d}\|^{2}} .
$$

\section{APPENDIX E}

\section{DERIVATION OF THE OPTIMAL MMSE EQUALIZER WEIGHT}

The equalization error at the $m$-th frequency tone is defined as the difference between the equalized observation and signal in the frequency domain, given by

$$
\varepsilon_{m}=W_{m} Z_{m}-X_{m},
$$

and the optimal weight at the $m$-th frequency tone based on MMSE criterion, i.e., $W_{\text {MMSE-opt }, m}$, minimizes the mean square error as follows,

$$
W_{\text {MMSE-opt }, m}=\arg \min _{W_{m}} \mathbb{E}\left[\left|\varepsilon_{m}\right|^{2}\right],
$$

which is equivalent to find the $W_{m}$ satisfying

$$
\frac{\partial \mathbb{E}\left[\left|\varepsilon_{m}\right|^{2}\right]}{\partial W_{m}}=0
$$

According to (66), the mean square error has its expansion on top of the next page as (69). Substituting (69) into (68), the solution is given by (70), where the relevant components are derived as follows. 


$$
\begin{aligned}
\mathbb{E}\left[\left|\varepsilon_{m}\right|^{2}\right]= & \mathbb{E}\left[\left(W_{m} Z_{m}-X_{m}\right)\left(W_{m} Z_{m}-X_{m}\right)^{*}\right] \\
= & \mathbb{E}\left[W_{m} W_{m}^{*}\left|Z_{m}\right|^{2}-W_{m} Z_{m} X_{m}^{*}-Z_{m}^{*} W_{m}^{*} X_{m}+\left|X_{m}\right|^{2}\right] \\
= & W_{m} W_{m}^{*}\left(\left|H_{m}\right|^{2} \mathbb{E}\left[\left|X_{m}\right|^{2}\right]+H_{m} \mathbb{E}\left[X_{m} N_{m}^{*}\right]+H_{m}^{*} \mathbb{E}\left[X_{m}^{*} N_{m}\right]+\mathbb{E}\left[\left|N_{m}\right|^{2}\right]\right) \\
& -W_{m}\left(H_{m} \mathbb{E}\left[\left|X_{m}\right|^{2}\right]+\mathbb{E}\left[X_{m}^{*} N_{m}\right]\right)-W_{m}^{*}\left(H_{m}^{*} \mathbb{E}\left[\left|X_{m}\right|^{2}\right]+\mathbb{E}\left[X_{m} N_{m}^{*}\right]\right)+\mathbb{E}\left[\left|X_{m}\right|^{2}\right] \\
& W_{\text {MMSE-opt }, m}=\frac{H_{m}^{*} \mathbb{E}\left[\left|X_{m}\right|^{2}\right]+\mathbb{E}\left[X_{m} N_{m}^{*}\right]}{\left|H_{m}\right|^{2} \mathbb{E}\left[\left|X_{m}\right|^{2}\right]+H_{m}^{*} \mathbb{E}\left[X_{m}^{*} N_{m}\right]+H_{m} \mathbb{E}\left[X_{m} N_{m}^{*}\right]+\mathbb{E}\left[\left|N_{m}\right|^{2}\right]}
\end{aligned}
$$

According to the results in (31) and (36), we have

$$
\left|X_{m}\right|^{2}=\frac{Q^{2}}{M} \sum_{k=0}^{M-1} \sum_{l=0}^{M-1} b_{k} b_{l} \exp \left(\frac{j 2 \pi m(l-k)}{M}\right) .
$$

Based on (10), the random variable $b_{k} \sim \operatorname{Bernoulli}(p)$, where $p$ is the probability for bit- 1 , and $p=0.5$ is a valid assumption for consecutive transmission with a long data length. In light of this, we have

$$
\mathbb{E}\left[b_{k} b_{l}\right]= \begin{cases}\frac{1}{2}, & k=l, \\ \frac{1}{4}, & \text { otherwise. }\end{cases}
$$

According to the results of (72), the expectation form of (71) is given by

$$
\begin{aligned}
\mathbb{E}\left[\left|X_{m}\right|^{2}\right] & =\frac{Q^{2}}{M} \sum_{k=0}^{M-1} \sum_{l=0}^{M-1} \mathbb{E}\left[b_{k} b_{l}\right] \exp \left(\frac{j 2 \pi m(l-k)}{M}\right) \\
& =\frac{Q^{2}}{2}+\frac{Q^{2}}{4 M} \sum_{k=0}^{M-1} \sum_{l \neq k} \exp \left(\frac{j 2 \pi m(l-k)}{M}\right) .
\end{aligned}
$$

In a similar way, from the result given in (37), we can obtain

$$
\left|N_{m}\right|^{2}=\frac{1}{M} \sum_{k=0}^{M-1} \sum_{l=0}^{M-1} n_{k} n_{l} \exp \left(\frac{j 2 \pi m(l-k)}{M}\right) .
$$

Then, based on (32), the expectation form of (74) is given by

$$
\mathbb{E}\left[\left|N_{m}\right|^{2}\right]=\frac{1}{M} \sum_{k=0}^{M-1} \mathbb{E}\left[n_{k}^{2}\right]=\frac{1}{M V_{\mathrm{R}}} \sum_{k=0}^{M-1} y_{k} .
$$

In addition, we have

$$
X_{m} N_{m}^{*}=\frac{Q}{M} \sum_{k=0}^{M-1} \sum_{l=0}^{M-1} b_{k} n_{l} \exp \left(\frac{j 2 \pi m(l-k)}{M}\right)
$$

and we have

$$
\mathbb{E}\left[b_{k} n_{l}\right]=\mathbb{E}_{b_{k}}\left[b_{k} \mathbb{E}_{n_{l} \mid b_{k}}\left[n_{l}\right]\right]=0 .
$$

According to (77), expectation of (76) is

$\mathbb{E}\left[X_{m} N_{m}^{*}\right]=\frac{Q}{M} \sum_{k=0}^{M-1} \sum_{l=0}^{M-1} \mathbb{E}\left[b_{k} n_{l}\right] \exp \left(\frac{j 2 \pi m(l-k)}{M}\right)=0$

and its conjugate form can be given by

$$
\mathbb{E}\left[X_{m}^{*} N_{m}\right]=\mathbb{E}\left[X_{m} N_{m}^{*}\right]^{*}=0 .
$$

Substituting (78) and (79) into (69), the optimal MMSE equalizer weight can be simplified to

$$
W_{\text {MMSE-opt }, m}=\frac{H_{m}^{*}}{\left|H_{m}\right|^{2}+\frac{\mathbb{E}\left[\left|N_{m}\right|^{2}\right]}{\mathbb{E}\left[\left|X_{m}\right|^{2}\right]}} .
$$

\section{REFERENCES}

[1] T. Nakano, A. W. Eckford, and T. Haraguchi, Molecular Communication. Cambridge University Press, 2013.

[2] Y. Lu, R. Ni, and Q. Zhu, "Wireless communication in nanonetworks: Current status, prospect and challenges," IEEE Trans. Mol. Biol. MultiScale Commun., vol. 6, no. 2, pp. 71-80, Nov. 2020.

[3] N. Farsad et al., "A comprehensive survey of recent advancements in molecular communication," IEEE Commun. Survey \& Tut., vol. 18, no. 3, pp. 1887-1919, third quarter 2016.

[4] M. U. Mahfuz, D. Makrakis, and H. T. Mouftah, "On the characterization of binary concentration-encoded molecular communication in nanonetworks," Nano Commun. Netw., vol. 1, no. 4, pp. 289-300, Dec. 2010.

[5] M. S. Kuran, H. B. Yilmaz, T. Tugcu, and I. F. Akyildiz, "Modulation techniques for communication via diffusion in nanonetworks," in Proc. IEEE International Conf. on Commun. (ICC), Kyoto, Japan, June 2011, pp. 1-5.

[6] L. Shi and L. L. Yang, "Error performance analysis of diffusive molecular communication systems with on-off keying modulation," IEEE Trans. Mol. Biol. Multi-Scale Commun., vol. 3, no. 4, pp. 224-238, Dec. 2017.

[7] N. Kim and C. Chae, "Novel modulation techniques using isomers as messenger molecules for nano communication networks via diffusion," IEEE J. Sel. Areas Commun., vol. 31, no. 12, pp. 847-856, Dec. 2013.

[8] X. Chen, Y. Huang, L. L. Yang, and M. Wen, "Generalized molecularshift keying (GMoSK): Principles and performance analysis," IEEE Trans. Mol. Biol. Multi-Scale Commun., vol. 6, no. 3, pp. 168-183, Dec. 2020.

[9] Y. Tang, M. Wen, X. Chen, Y. Huang, and L. L. Yang, "Molecular type permutation shift keying for molecular communication," IEEE Trans. Mol. Biol. Multi-Scale Commun., vol. 6, no. 2, pp. 160-164, Nov. 2020.

[10] L. Meng, P. Yeh, K. Chen, and I. F. Akyildiz, "MIMO communications based on molecular diffusion," in Proc. IEEE Global Commun. Conf. (GLOBECOM), Anaheim, CA, USA, Dec. 2012, pp. 5380-5385.

[11] B. Koo, C. Lee, H. B. Yilmaz, N. Farsad, A. Eckford, and C. Chae, "Molecular MIMO: From theory to prototype," IEEE J. Sel. Areas Commun., vol. 34, no. 3, pp. 600-614, Mar. 2016.

[12] S. M. R. Rouzegar and U. Spagnolini, "Diffusive MIMO molecular communications: Channel estimation, equalization, and detection," IEEE Trans. Commun., vol. 67, no. 7, pp. 4872-4884, July 2019.

[13] Y. Huang, M. Wen, L. L. Yang, C. Chae, and F. Ji, "Spatial modulation for molecular communication," IEEE Trans. Nanobiosci., vol. 18, no. 3, pp. 381-395, July 2019.

[14] M. C. Gursoy, E. Basar, A. E. Pusane, and T. Tugcu, "Index modulation for molecular communication via diffusion systems," IEEE Trans. Commun., vol. 67, no. 5, pp. 3337-3350, May 2019.

[15] M. S. Kuran, H. B. Yilmaz, I. Demirkol, N. Farsad, and A. Goldsmith, "A survey on modulation techniques in molecular communication via diffusion," IEEE Commun. Survey \& Tut., pp. 1-1, 2020.

[16] M. S. Kuran, H. B. Yilmaz, T. Tugcu, and I. F. Akyildiz, "Interference effects on modulation techniques in diffusion based nanonetworks," Nano Commun. Netw., vol. 3, no. 1, pp. 65-73, Mar. 2012.

[17] L. Meng, P. Yeh, K. Chen, and I. F. Akyildiz, "On receiver design for diffusion-based molecular communication," IEEE Trans. Signal Process., vol. 62, no. 22, pp. 6032-6044, Nov. 2014. 
[18] D. Kilinc and O. B. Akan, "Receiver design for molecular communication," IEEE J. Sel. Areas Commun., vol. 31, no. 12, pp. 705-714, Dec. 2013.

[19] M. S. Thakur, S. Jain, and V. Bhatia, "Volterra-DFE based nonlinear equalizer for diffusion based molecular communications," in Proc. IEEE International Conf. on Advanced Netw. and Telecommun. Syst. (ANTS), GOA, India, Dec. 2019, pp. 1-5.

[20] T. N. Cao, V. Jamali, N. Zlatanov, P. L. Yeoh, J. Evans, and R. Schober, "Fractionally spaced equalization and decision feedback sequence detection for diffusive MC,' IEEE Commun. Lett., vol. 25, no. 1, pp. 117-121, Jan. 2021.

[21] M. C. Gursoy, A. Celik, E. Basar, A. E. Pusane, and T. Tugcu, "Molecular index modulation with space-time equalization," IEEE Wireless Commun. Lett., vol. 9, no. 5, pp. 702-705, May 2020.

[22] L. Shi and L. L. Yang, "Equalisation and performance of diffusive molecular communication systems with binary molecular-shift keying modulation," IET Commun., vol. 14, no. 4, pp. 549-555, Mar. 2020.

[23] A. Sohail and Y. Sanada, "Novel frequency domain equalization with threshold for molecular communication," IEICE Commun. Express, vol. 9, no. 2, pp. 36-41, 2020.

[24] — - "Novel approximated zero forcing pre-coding technique with threshold for diffusion based molecular communication," IEICE Commun. Express, vol. 9, no. 7, pp. 348-353, 2020.

[25] M. Pierobon and I. F. Akyildiz, "A physical end-to-end model for molecular communication in nanonetworks," IEEE J. Sel. Areas Commun., vol. 28, no. 4, pp. 602-611, May 2010.

[26] _ - "Diffusion-based noise analysis for molecular communication in nanonetworks," IEEE Trans. Signal Process., vol. 59, no. 6, pp. 2532 2547, June 2011.

[27] N. Garralda, I. Llatser, A. Cabellos-Aparicio, E. Alarcn, and M. Pierobon, "Diffusion-based physical channel identification in molecular nanonetworks," Nano Commun. Netw., vol. 2, no. 4, pp. 196-204, Dec. 2011.

[28] S. Wang, W. Guo, and M. D. McDonnell, "Transmit pulse shaping for molecular communications," in Proc. IEEE Conf. on Comput. Commun. Workshops (INFOCOM WKSHPS), Toronto, Canada, Apr. 2014, pp. 209-210.

[29] M. J. Berridge, "The AM and FM of calcium signalling," Nature, vol. 386, p. 759760, Apr. 1997.

[30] C. T. Chou, "Molecular circuits for decoding frequency coded signals in nano-communication networks," Nano Commun. Netw., vol. 3, no. 1, pp. 46-56, Mar. 2012.

[31] W. Guo, B. Li, S. Wang, and W. Liu, "Molecular communications with longitudinal carrier waves: Baseband to passband modulation," IEEE Commun. Lett., vol. 19, no. 9, pp. 1512-1515, Sept. 2015.

[32] M. Damrath, J. J. Koshy, and P. A. Hoeher, "Application of OFDM in diffusion-based molecular communication," IEEE Trans. Mol. Biol. Multi-Scale Commun., vol. 3, no. 4, pp. 254-258, Dec. 2017.

[33] B. Li, W. Guo, X. Wang, Y. Deng, Y. Lan, C. Zhao, and A. Nallanathan, "CSI-independent non-linear signal detection in molecular communications," IEEE Trans. Signal Process., vol. 68, pp. 97-112, Jan. 2020.

[34] M. Khalid, O. Amin, S. Ahmed, B. Shihada, and M. Alouini, "Modeling of viral aerosol transmission and detection," IEEE Trans. Commun. vol. 68, no. 8, pp. 4859-4873, Aug. 2020.

[35] P. He, T. Nakano, Y. Mao, P. Lio, Q. Liu, and K. Yang, "Stochastic channel switching of frequency-encoded signals in molecular communication networks," IEEE Commun. Lett., vol. 22, no. 2, pp. 332-335, Feb. 2018

[36] V. Jamali, A. Ahmadzadeh, W. Wicke, A. Noel, and R. Schober, "Channel modeling for diffusive molecular communicationa tutorial review," Proc. IEEE, vol. 107, no. 7, pp. 1256-1301, July 2019.

[37] A. Noel, K. C. Cheung, and R. Schober, "Using dimensional analysis to assess scalability and accuracy in molecular communication," in Proc. IEEE International Conf. on Commun. Workshops (ICC), Budapest, Hungary, June 2013, pp. 818-823.

[38] F. Amoroso, "The bandwidth of digital data signal," IEEE Commun. Mag., vol. 18, no. 6, pp. 13-24, Nov. 1980.

[39] F. Chapeau-Blondeau and A. Monir, "Numerical evaluation of the lambert $\mathrm{W}$ function and application to generation of generalized gaussian noise with exponent 1/2," IEEE Trans. Signal Process., vol. 50, no. 9, pp. 2160-2165, Sept. 2002.

[40] M. J. Moore, T. Nakano, A. Enomoto, and T. Suda, "Measuring distance from single spike feedback signals in molecular communication," IEEE Trans. Signal Process., vol. 60, no. 7, pp. 3576-3587, July 2012.

[41] F. Pancaldi, G. M. Vitetta, R. Kalbasi, N. Al-Dhahir, M. Uysal, and H. Mheidat, "Single-carrier frequency domain equalization," IEEE Signal Process. Mag., vol. 25, no. 5, pp. 37-56, Sept. 2008.
[42] H. ShahMohammadian, G. G. Messier, and S. Magierowski, "Blind synchronization in diffusion-based molecular communication channels," IEEE Commun. Lett., vol. 17, no. 11, pp. 2156-2159, Nov. 2013.

[43] L. Lin, C. Yang, M. Ma, S. Ma, and H. Yan, "A clock synchronization method for molecular nanomachines in bionanosensor networks," IEEE Sensors J., vol. 16, no. 19, pp. 7194-7203, Oct. 2016.

[44] L. Lin, J. Zhang, M. Ma, and H. Yan, "Time synchronization for molecular communication with drift," IEEE Commun. Lett., vol. 21, no. 3, pp. 476-479, Mar. 2017.

[45] V. Jamali, A. Ahmadzadeh, and R. Schober, "Symbol synchronization for diffusion-based molecular communications," IEEE Trans. Nanobiosci., vol. 16, no. 8, pp. 873-887, Dec. 2017.

[46] T. Tung and U. Mitra, "Synchronization error robust transceivers for molecular communication," IEEE Trans. Mol. Biol. Multi-Scale Commun., vol. 5, no. 3, pp. 207-221, Dec. 2019.

[47] V. Jamali, A. Ahmadzadeh, C. Jardin, H. Sticht, and R. Schober, "Channel estimation for diffusive molecular communications," IEEE Trans. Commun., vol. 64, no. 10, pp. 4238-4252, Oct. 2016.

[48] V. Jamali, N. Farsad, R. Schober, and A. Goldsmith, "Non-coherent detection for diffusive molecular communication systems," IEEE Trans. Commun., vol. 66, no. 6, pp. 2515-2531, June 2018.

[49] S. Abdallah and A. M. Darya, "Semi-blind channel estimation for diffusive molecular communication," IEEE Commun. Lett., vol. 24, no. 11, pp. 2503-2507, Nov. 2020.

[50] Y. Tang, Y. Huang, C. B. Chae, W. Duan, M. Wen, and L. L. Yang, "Molecular type permutation shift keying in molecular mimo communications for iobnt," IEEE Internet Things J., pp. 1-1, 2021.

[51] F. Adachi, H. Tomeba, and K. Takeda, "Introduction of frequencydomain signal processing to broadband single-carrier transmissions in a wireless channel," IEICE Trans. Commun., vol. E92.B, no. 9, pp. 27892808, Sept. 2009.

[52] J. Coon, S. Armour, M. Beach, and J. McGeehan, "Adaptive frequencydomain equalization for single-carrier multiple-input multiple-output wireless transmissions," IEEE Trans. Signal Process., vol. 53, no. 8, pp. 3247-3256, Aug. 2005.

[53] H. Yan, G. Chang, Z. Ma, and L. Lin, "Derivative-based signal detection for high data rate molecular communication system," IEEE Commun. Lett., vol. 22, no. 9, pp. 1782-1785, Sept. 2018.

[54] Y. Huang, X. Chen, M. Wen, L. L. Yang, C. Chae, and F. Ji, "A rising edge-based detection algorithm for MIMO molecular communication," IEEE Wireless Commun. Lett., vol. 9, no. 4, pp. 523-527, Apr. 2020.

[55] B. Li, M. Sun, S. Wang, W. Guo, and C. Zhao, "Local convexity inspired low-complexity noncoherent signal detector for nanoscale molecular communications," IEEE Trans. Commun., vol. 64, no. 5, pp. 2079-2091, May 2016.

[56] Z. Wei, W. Guo, B. Li, J. Charmet, and C. Zhao, "High-dimensional metric combining for non-coherent molecular signal detection," IEEE Trans. Commun., vol. 68, no. 3, pp. 1479-1493, Mar. 2020.

[57] N. Farsad and A. Goldsmith, "Neural network detection of data sequences in communication systems," IEEE Trans. Signal Process., vol. 66 , no. 21 , pp. 5663-5678, Nov. 2018.

[58] C. Toker and G. Altin, "Blind, adaptive channel shortening equalizer algorithm which can provide shortened channel state information (bacssi)," IEEE Trans. Signal Process., vol. 57, no. 4, pp. 1483-1493, Apr. 2009.

[59] D. Darsena, G. Gelli, L. Paura, and F. Verde, "Blind channel shortening for space-time-frequency block coded MIMO-OFDM systems," IEEE Trans. Wireless Commun., vol. 11, no. 3, pp. 1022-1033, Mar. 2012.

[60] M. S. Thakur, S. Sharma, and V. Bhatia, "Molecular signal detection using nonlinear mapping," IEEE Wireless Commun. Lett., pp. 1-1, 2020.

[61] F. W. J. Olver, D. W. Lozier, R. F. Boisvert, and C. W. Clark, NIST Handbook of Mathematical Functions. Cambridge University Press, 2010. 


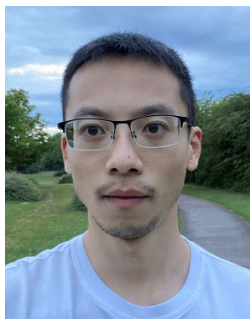

Yu Huang (S'19) received the B.S. degree in communication engineering from the Harbin University of Science and Technology, Harbin, China, in 2016. $\mathrm{He}$ is currently pursuing the Ph.D. degree in information and communication engineering with the South China University of Technology, Guangzhou, China. During the Summer vacations of 2018 and 2019, he was a visiting student of molecular communication with Yonsei University, Seoul, South Korea. From January 2020 to March 2021, he visited Cranfield University, Bedford, U.K., for emerging communication and artificial intelligence techniques. His main research interests include molecular communications and wireless communications.

He was the winner of the Data Bakeoff Competition (Molecular MIMO) at the IEEE Communication Theory Workshop held in Selfoss, Iceland, in 2019.

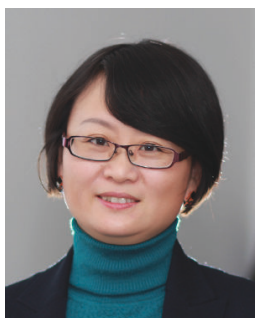

Fei Ji (M'06) received the B.S. degree in applied electronic technologies from Northwestern Polytechnical University, Xian, China, and the M.S. degree in bioelectronics and Ph.D. degree in circuits and systems both from the South China University of Technology, Guangzhou, China, in 1992, 1995, and 1998 , respectively. She was a Visiting Scholar with the University of Waterloo, Canada, from June 2009 to June 2010. She worked in the City University of Hong Kong as a Research Assistant from March 2001 to July 2002 and a Senior Research Associate from January 2005 to March 2005. She is currently a Professor with the School of Electronic and Information Engineering, South China University of Technology. She was the Registration Chair and the Technical Program Committee (TPC) member of IEEE 2008 International Conference on Communication System. Her research focuses on wireless communication systems and networking.

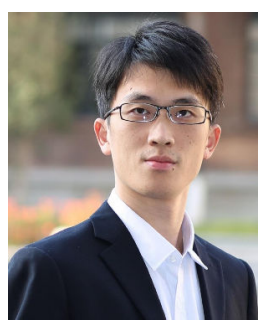

Miaowen Wen (SM18) received the Ph.D. degree from Peking University, Beijing, China, in 2014 From 2012 to 2013, he was a Visiting Student Research Collaborator with Princeton University, Princeton, NJ, USA. He is currently an Associate Professor with South China University of Technology, Guangzhou, China. He has published two books and more than 100 journal papers. His research interests include a variety of topics in the areas of wireless and molecular communications.

Dr. Wen was a recipient of the IEEE Asia-Pacific (AP) Outstanding Young Researcher Award in 2020, and four Best Paper Awards from the IEEE ITST12, the IEEE ITSC14, the IEEE ICNC16, and the IEEE ICCT19. He was the winner in data bakeoff competition (Molecular MIMO) at IEEE Communication Theory Workshop (CTW) 2019, Selfoss, Iceland. He served as a Guest Editor for the IEEE JOURNAL ON SELECTED AREAS IN COMMUNICATIONS and for the IEEE JOURNAL OF SELECTED TOPICS IN SIGNAL PROCESSING. Currently, he is serving as an Editor for the IEEE TRANSACTIONS ON COMMUNICATIONS, and the IEEE COMMUNICATIONS LETTERS, and a Guest Editor for the IEEE JOURNAL OF SELECTED TOPICS IN SIGNAL PROCESSING (Special Issue on Advanced Signal Processing for Local and Private 5G Networks).

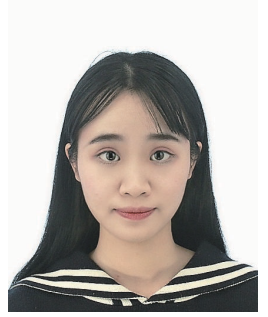

Xuan Chen (S'19) received the B.S. degree from Wuhan University of Technology, Wuhan, China, in 2017. She is currently pursuing the Ph.D. degree with the South China University of Technology, Guangzhou, China. In August 2019, she was a visiting student for molecular communication in Yonsei University, Seoul, South Korea. Her main research interest includes emerging technologies for $6 \mathrm{G}$ communications.

She was the winner in data bakeoff competition Workshop (CTW) 2019, Selfoss, Iceland.

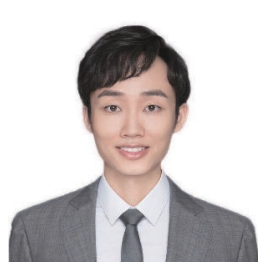

Yuankun Tang (S'19) received the B.S. degree in communication engineering from the South China University of Technology, Guangzhou, China, in 2018. He is currently pursuing the Ph.D. degree in information and communication engineering with the South China University of Technology, Guangzhou, China. In August 2019, he was a visiting student of molecular communication with Yonsei University, Seoul, South Korea. His main research interests include molecular communications and index modulation.

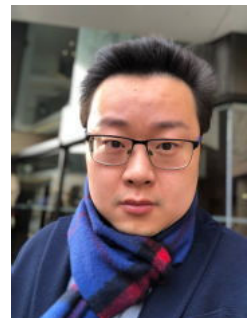

Weisi Guo (S'07, M'11, SM'17) received his MEng, $\mathrm{MA}$, and Ph.D. degrees from the University of Cambridge, UK. He is Chair Professor of Human Machine Intelligence at Cranfield University. He has published over 180 papers and is PI on a number of molecular communication research grants. His research has won him several international awards (IET Innovation 15, Bell Labs Prize Finalist 14, 16 , and 19). He was a Turing Fellow at the Alan Turing Institute and Fellow of Royal Statistical Society.

Zhuangkun Wei received his Ph.D. degree from the School of Engineering, The University of Warwick, UK in 2021, and Master's \& Bachelors degrees in Electronic Engineering from Beijing University of Posts and Telecommunications (BUPT), Beijing, China in 2018 and 2014 respectively. He is currently a research fellow in Secure Trustworthy Autonomous Systems in the School of Aerospace, Transport and Manufacturing, Cranfield University. His research interests cover physical layer security, graph signal processing, and molecular communica- 
2021-03-17

Frequency domain analysis and equalization for molecular communication

Huang, Yu

IEEE

Huang Y, Ji F, Wei Z, et al., (2021) Frequency domain analysis and equalization for molecular communication. IEEE Transactions on Signal Processing, Volume 69, pp. 1952 - 1967

https://doi.org/10.1109/TSP.2021.3066069

Downloaded from Cranfield Library Services E-Repository 